\title{
From evolved stars to the evolution of IC 1613
}

\author{
Seyed Azim Hashemi, ${ }^{1,2 \star}$ Atefeh Javadi ${ }^{2 \star}$ and Jacco Th. van Loon ${ }^{3}$ \\ ${ }^{1}$ Department of Physics, Sharif University of Technology, Tehran 11155-9161, Iran \\ ${ }^{2}$ School of Astronomy, Institute for Research in Fundamental Sciences (IPM), Tehran 19395-5531, Iran \\ ${ }^{3}$ Lennard-Jones Laboratories, Keele University, Staffordshire ST5 5BG, UK
}

Accepted 2018 December 17. Received 2018 December 17; in original form 2018 March 11

\begin{abstract}
IC 1613 is a Local Group dwarf irregular galaxy at a distance of $750 \mathrm{kpc}$. In this work, we present an analysis of the star formation history (SFH) of a field of $\sim 200 \mathrm{arcmin}^{2}$ in the central part of the galaxy. To this aim, we use a novel method based on the resolved population of more highly evolved stars. We identify 53 such stars, eight of which are supergiants and the remainder are long-period variables, large-amplitude variables, or extreme asymptotic giant branch (x-AGB) stars. Using stellar evolution models, we find the age and birth mass of these stars and thus reconstruct the SFH. The average rate of star formation during the last Gyr is $\sim 3 \times 10^{-4} \mathrm{M}_{\odot} \mathrm{yr}^{-1} \mathrm{kpc}^{-2}$. The absence of a dominant epoch of star formation over the past 5 Gyr suggests that IC 1613 has evolved in isolation for that long, spared harassment by other Local Group galaxies (in particular M 31 and the Milky Way). We confirm the radial age gradient, with star formation currently concentrated in the central regions of IC 1613, and the failure of recent star formation to have created the main H I supershell. Based on the current rate of star formation at $(5.5 \pm 2) \times 10^{-3} \mathrm{M}_{\odot} \mathrm{yr}^{-1}$, the interstellar gas mass of the galaxy of $9 \times 10^{7} \mathrm{M}_{\odot}$, and the gas production rate from AGB stars at $\sim 6 \times 10^{-4} \mathrm{M}_{\odot} \mathrm{yr}^{-1}$, we conclude that the star formation activity of IC 1613 can continue for $\sim 18 \mathrm{Gyr}$ in a closed-box model, but is likely to cease much earlier than that unless gas can be accreted from outside.
\end{abstract}

Key words: stars: $\mathrm{AGB}$ and post-AGB -supergiants-stars: variables: general-galaxies: dwarf-galaxies: evolution-galaxies: individual: IC 1613.

\section{INTRODUCTION}

The most abundant type of galaxy in the Local Group are low-mass dwarf galaxies and according to popular structure formation theory (e.g. White \& Rees 1978; Blumenthal et al. 1984; White \& Frenk 1991; Mo, van den Bosch \& White 2010) we expect that this holds true for the whole Universe both in space and time. Their vicinity allows us access to their structure, star formation history (SFH), and chemical composition, all of which are the result of galaxy formation and evolution (e.g. Hodge 1989; Chun et al. 2015). Unlike their massive siblings, dwarf galaxies are readily disturbed via interactions with larger galaxies and the intracluster medium, as well as internal processes that remove gas from these loosely bound systems (Stinson et al. 2007). Proximity and simplicity of dwarf galaxies make them superb test cases to probe the effect of different mechanisms operating in the internal and external evolution of galaxies. Determining the SFH has a key role in this. In this work, we set out to determine the SFH of IC 1613, an isolated dwarf irreg-

^ E-mail: hashemi_seyedazim@physics.sharif.edu (SAH); atefeh@ipm.ir $(\mathrm{AJ})$ ular galaxy, and to use it to answer questions about its interaction history, stellar population age gradient, and morphology.

Much of the history of galaxies is imprinted upon the most highly evolved stellar populations, spanning lookback times from $10^{7}$ to $10^{10} \mathrm{yr}$. The high luminosity of $\sim 2000 \mathrm{~L}_{\odot}$ for tip of the red-giant branch (TRGB) stars, $\sim 10^{4} \mathrm{~L}_{\odot}$ for asymptotic giant branch (AGB) stars, and a few $10^{5} \mathrm{~L}_{\odot}$ for red supergiants (RSGs) make cool evolved stars one of the most accessible probes of the underlying stellar populations in the infrared (IR; e.g. Maraston 2005; Maraston et al. 2006). Their spectral energy distributions (SEDs) peak around $1 \mu \mathrm{m}$, so they stand out in the near-IR, where extinction and reddening by dust are relatively low. They have low surface gravity causing them to pulsate radially on time-scales of a few weeks to a few years. The long-period variable (LPV) stars are typically AGB stars in the final stages of evolution (Fraser et al. 2005). All of the thermally pulsing AGB (TP-AGB) stars are LPVs (e.g. Fraser, Hawley \& Cook 2008; Soszyński et al. 2009), with periods of $>100$ d (e.g. Iben \& Renzini 1983; Padova evolutionary tracks - Marigo et al. 2008). Furthermore, there is a good correlation between increasing period and increasing amplitude (Fraser et al. 2008) and mass loss (Goldman et al. (2017), and large-amplitude variable (LAV) stars therefore also bear a strong relation to the end points of stellar evolution. In the 
absence of period determinations, selection on the basis of amplitude would also result in samples of the most highly evolved AGB stars.

In the AGB phase, the rate of mass loss accelerates with time. This is because the luminosity and radius are increasing while the mass is decreasing, thus leading to reduced surface gravity and less strongly bound surface layers. In the final phase, a 'superwind' develops characterized by the highest dust fraction (e.g. Schröder, Winters \& Sedlmayr 1999; Lagadec \& Zijlstra 2008). Hence, very dusty AGB stars (extreme AGB stars, or x-AGB) are at the end of their evolution on the AGB (e.g. Groenewegen et al. 1998; Carroll \& Ostlie 2007).

While AGB stars originating from solar-mass stars have ages of $\sim 10 \mathrm{Gyr}$, the most luminous AGB stars formed only $\sim 10^{8} \mathrm{yr}$ ago. To probe more recently formed stellar populations, we include RSGs in our analysis, which can be as young as $10^{7} \mathrm{yr}$ old. The coolest RSGs also pulsate with long periods and (in energy terms) considerable amplitude (van Loon et al. 2008). We have developed a novel method to use the relative numbers of these evolved AGB stars and RSGs and their lifetimes to reconstruct the SFH (Javadi, van Loon \& Mirtorabi 2011; Rezaeikh et al. 2014; Golshan et al. 2017; Javadi et al. 2017). In the following, we will apply this technique to IC 1613.

IC 1613 is an isolated dwarf galaxy within the Local Group, discovered by Wolf (1906). We adopt the mean distance of $750 \mathrm{kpc}$ $\left((m-M)_{0}=24.37 \pm 0.08 \mathrm{mag}\right)$ from Menzies, Whitelock \& Feast (2015). Its vicinity, inclination angle $\left(i=38^{\circ}\right.$; Lake \& Skillman $1989)$, and low foreground reddening $(E(B-V)=0.025 \mathrm{mag}$; Schlegel, Finkbeiner \& Davis 1998) make it a target of choice for the study of its stellar populations. Its stellar mass is estimated to be $\sim 2 \times 10^{8} \mathrm{M}_{\odot}$ (see Dekel \& Woo 2003; Orban et al. 2008), which is similar to its dynamical mass of $(1.1 \pm 0.2) \times$ $10^{8} \mathrm{M}_{\odot}$ estimated by Kirby et al. (2014) (the observed maximum rotation velocity is $25 \mathrm{~km} \mathrm{~s}^{-1}$; Lake \& Skillman 1989) suggesting no significant dark matter within the optical half-light radius $(\sim 1.4 \mathrm{kpc})$.

The history of IC 1613 is principally enshrined in its SFH. Cole et al. (1999) estimated a roughly constant star formation rate (SFR) across the central $0.22 \mathrm{kpc}^{2}$ during the past $250-350 \mathrm{Myr}$, but 50 per cent higher $400-900 \mathrm{Myr}$ ago. The SFR in the central part over the past $300 \mathrm{Myr}$ was estimated by Bernard et al. (2007) to be $(1.6 \pm 0.8) \times 10^{-3} \mathrm{M}_{\odot} \mathrm{yr}^{-1} \mathrm{kpc}^{-2}$. Skillman et al. (2014), on the other hand, found that the SFR in a small field near the halflight radius of IC 1613 has been constant over the entire lifetime of IC 1613.

Chemical evolution places additional constraints on the history of a galaxy, with the overall metallicity increasing in time as subsequent generations of stars synthesize metals and return them to the interstellar medium (ISM). Cole et al. (1999) found that the metallicity of IC 1613 is comparable to that of the Small Magellanic Cloud - remarkable given the latter is 10 times more massive. Dolphin et al. (2001) derived a mean value of $[\mathrm{Fe} / \mathrm{H}]=-1.15 \pm 0.2 \mathrm{dex}$ $(Z \sim 0.001)$, while Tikhonov \& Galazutdinova (2002) derived a lower value of $[\mathrm{Fe} / \mathrm{H}]=-1.75 \pm 0.2 \mathrm{dex}(Z \sim 0.0003)$ for the old population, the youngest population is expected to be more metal rich. Indeed, Skillman, Côté \& Miller (2003) found that [Fe/H] has increased from $-1.3 \mathrm{dex}(Z \sim 0.0008)$ at early times to $-0.7 \mathrm{dex}$ $(Z \sim 0.003)$ at present, which is confirmed by Tautvaišienè et al.'s (2007) estimate of $[\mathrm{Fe} / \mathrm{H}]=-0.67 \mathrm{dex}(Z \sim 0.003)$ for the young population.

The structure of this paper is as follows. In Section 2, we describe the data we use. In Section 3, we introduce our method. In Section 4, we derive the $\mathrm{SFH}$, followed by a discussion and conclusions in Sections 5 and 6.

\section{DATA}

In this work, we benefit from a number of published data sets at near- and mid-IR wavelengths, which we shall describe in some detail below and which we summarize in Table 1 .

\subsection{Near-infrared data}

Menzies et al. (2015) published simultaneous $J H K_{\mathrm{s}}$ photometry from a 3-yr monitoring survey with the 1.4-m Infrared Survey Facility of the central $\sim 200 \mathrm{arcmin}^{2}$ region of IC 1613. They classified all objects brighter than the tip of the first ascent red giant branch (RGB; $K_{\mathrm{s}}=18 \mathrm{mag}$ ) as supergiants or AGB stars (but not foreground stars or background galaxies). They identified 758 variable stars with a standard error $<0.1 \mathrm{mag}$ in the $J H K_{\mathrm{s}}$ bands, and $10 \mathrm{ob}-$ jects that had already been known in the literature to be supergiants. Of these, 23 stars were identified as LAVs, for nine of which they determined periods of $>100 \mathrm{~d}$.

Sibbons et al. (2015) used the Wide Field Camera on the 3.8m United Kingdom Infrared Telescope to obtain $J H K$ photometry of a wider $0.8 \mathrm{deg}^{2}$ area centred on $\alpha=1^{\mathrm{h}} 4^{\mathrm{m}} 54^{\mathrm{s}}, \delta=2^{\circ} 7^{\prime} 57^{\prime \prime}$. Their catalogue presents dereddened photometry, listing 843 AGB stars within $4.5 \mathrm{kpc}$ from the centre of IC 1613. The TRGB was determined at $K=18.25 \pm 0.15 \mathrm{mag}$. From the colour separation between the carbon $(\mathrm{C})$ and $\mathrm{M}$-type stars among the AGB population at $(J-K)=1.15 \pm 0.05$ mag they determined a global $\mathrm{C} / \mathrm{M}$ ratio of 0.52 , and from this $[\mathrm{Fe} / \mathrm{H}]=-1.26 \pm 0.08 \mathrm{dex}(Z \sim 0.0008)$.

\subsection{Mid-infrared data}

AGB stars are cool and produce dust during the thermally pulsing phase. This dust makes them appear redder, so longer wavelength data are needed to identify the dustiest AGB stars that are in the final stages of evolution. Boyer et al. (2015a) observed IC 1613 as part of the DUST in Nearby Galaxies with Spitzer (DUSTiNGS) survey, using the Spitzer Space Telescope at 3.6 and $4.5 \mu \mathrm{m}$ to cover an area of $356 \mathrm{arcmin}^{2}$ on IC 1613 in two epochs, separated by $153 \mathrm{~d}$.

In the Large Magellanic Cloud, Blum et al. (2006) classified stars with $(J-[3.6])>3.1$ mag as 'extreme' AGB or X-AGB stars. Boyer et al. (2015b) devised a new criterion based solely on the Spitzer [3.6] and [4.5] bands, with a 93-94 percent success rate against using Blum's criterion in the Magellanic Clouds. Hence, among the 50 new variable AGB candidates that Boyer et al. detected, they identified $34 \mathrm{x}$-AGB candidates.

\subsection{Colour-magnitude diagrams}

Here we examine colour-magnitude diagrams (CMDs), in order to arrive at the currently best available sample of stars at their endpoints of evolution in the AGB phase, that we can use to derive the SFH. We follow a similar identification of features in the IR CMDs as in Blum et al. (2006), but using [4.5] instead of [8].

We cross-matched the stars from Sibbons et al. (2015) with stars in the 'good source catalogue' from Boyer et al. (2015a), using a matching radius of 1 arcsec. We thus identified 5788 stars in common, of which 750 are AGB stars. About 30 percent of Sibbons et al.'s sources were not matched with any of Boyer 
Table 1. Summary of photometric data.

\begin{tabular}{lccccccc}
\hline Telescope & $\begin{array}{c}\text { Photometric } \\
\text { bands }\end{array}$ & $\begin{array}{c}\text { Coverage } \\
\left(\operatorname{arcmin}^{2}\right)\end{array}$ & Total & AGB & LPV & x-AGB & \multicolumn{2}{c}{ Completeness limit } \\
$(\mathrm{mag})$
\end{tabular}
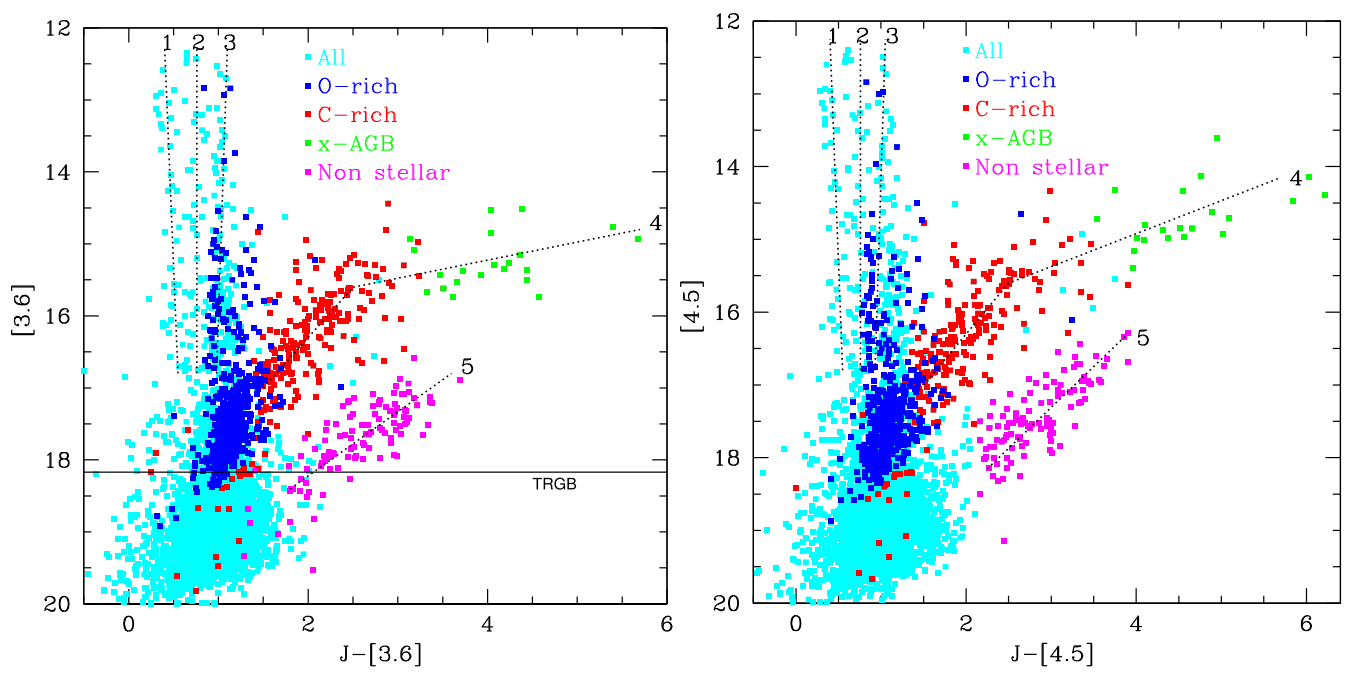

Figure 1. [3.6] versus $J-$ [3.6] (left) and [4.5] versus $J-$ [4.5] (right) colour-magnitude diagrams (CMDs). All cross-matched points are shown as cyan dots. Blue dots are oxygen-rich (O-rich) stars, and red dots are carbon-rich (C-rich) stars. The green dots represent x-AGB stars. The magenta dots are mainly background galaxies (see text). The solid line in the left-hand panel marks the tip of the red-giant branch (TRGB) at [3.6] = 18.17 mag (Boyer et al. 2009). Dashed numbered lines delineate sequences that we describe in the text.

et al.'s sources, which is largely a result of the different spatial coverage.

The [3.6] versus $J-[3.6]$ and [4.5] versus $J-[4.5]$ CMDs are presented in Fig. 1. Five sequences can be identified. The first of these, sequence $1(J-[3.6]<0.5 \mathrm{mag}$, reaching [3.6] $\sim 12 \mathrm{mag}$ ) corresponds to young A-G-type supergiants. Separated by a few tenths of magnitude to the red, sequence 2 consists mainly of foreground dwarfs and giants. Redder still by a similar amount, sequence 3 corresponds to RGB stars, AGB stars, and late-type (mostly M) supergiants in IC 1613 (Humphreys 1980; Herrero et al. 2010; Britavskiy et al. 2014; Menzies et al. 2015).

Sibbons et al. (2015) divided the region above the TRGB into Orich and C-rich where the latter have $(J-K)>1.15$ mag. In Fig. 1, the blue and red points correspond to Sibbons' photometric division of O-rich and C-rich, respectively, where the division occurs around $(J-[3.6]) \sim 1.4 \mathrm{mag}$ (similar for $J-[4.5])$. Of the 741 AGB stars from Sibbons et al. that have $J, H, K$, [3.6] and [4.5], we classed 477 ( $\sim 64$ per cent) as O-rich and $264(\sim 36$ per cent) as C-rich. This is very similar to Dell'Agli et al. (2016) where they compared models to the combination of near-IR (Sibbons et al. 2015) and mid-IR (Boyer et al. 2015a) data and found that the AGB sample of IC 1613 is composed of 65 percent O-rich and 35 per cent C-rich stars.

The C-stars (red) and x-AGB stars (green) form sequence 4. Up to $(J-[3.6]) \sim 3.1 \mathrm{mag}((J-[4.5]) \sim 3.5 \mathrm{mag})$ the sequence is dominated by stars that were classified from near-IR photometry as C-rich; beyond that is the realm of $\mathrm{x}$-AGB stars. We stress that while this sequence in other galaxies is normally dominated by carbon stars (Wood et al. 2011; Zijlstra et al. 2006) this does not preclude the presence of extremely dusty O-rich AGB stars among these red sources (van Loon et al. 1997). We classified all stars with $(J-[4.5])>3.5 \mathrm{mag}$ and [4.5] $<15.5 \mathrm{mag}$ as $\mathrm{x}$-AGB; these 21 objects are listed in Table 2. Of these x-AGB stars, all were found by Boyer et al. (2015b) to be variable (and x-AGB) at mid-IR wavelengths except \#7773 and \#7091 (from the Sibbons et al. 2015 catalogue), though Menzies et al. (2015) did identify \#7773 as a LAV.

Finally, sequence 5 (magenta in Fig. 1) is predominantly background galaxies (Meixner et al. 2006).

The numbers, and their contributions, of various populations identified in the CMDs are summarized in Table 3.

\subsection{Sample selection to determine the SFH}

Previous application of our method (Javadi et al. 2011, 2017; Rezaeikh et al. 2014; Golshan et al. 2017) - described in Section 3 was based on confirmed LPVs. LPVs are red giant or supergiant pulsating stars with periods ranging from months to a few years (e.g. Soszyński et al. 2009). In the case at hand, though, the limited cadency of the DUSTiNGS survey and the limited depth of the Menzies et al. (2015) survey will have led to LPVs being missed. Therefore, we combine confirmed LPVs with those AGB and RSG candidate stars that are also expected to be LPVs. These candidates are as follows. 
Table 2. Properties of the x-AGB stars identified in IC 1613.

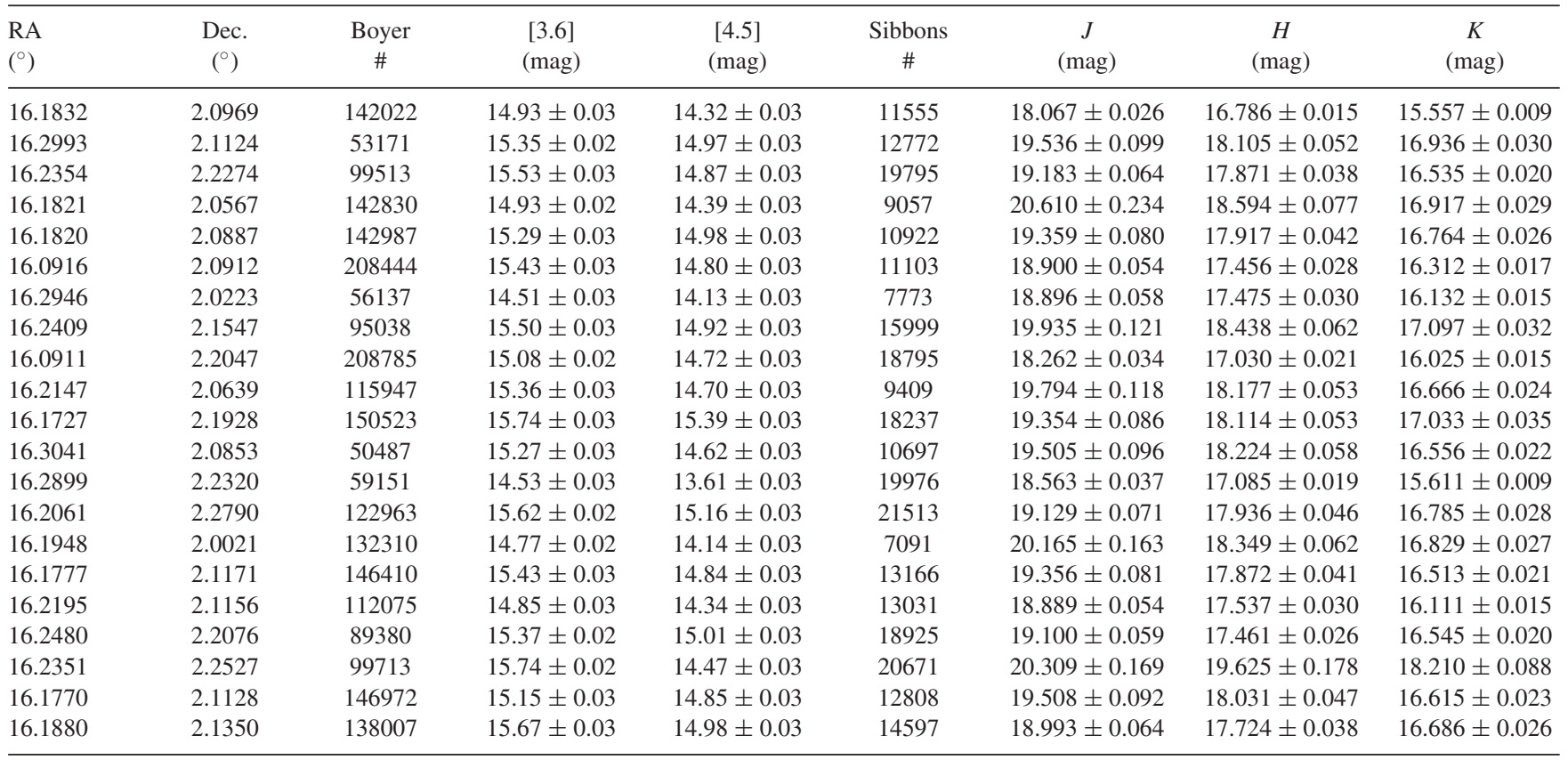

Table 3. Summary of CMD selections of stellar populations and colour plotted in Fig. 1.

\begin{tabular}{lcccc}
\hline Population & Diagram & $N$ & $\%$ & Colour \\
\hline Sources with $J$ and & {$[3.6], J-[3.6]$} & 5261 & 100 & Cyan \\
[3.6] & & & & \\
AGB with $J$ and [3.6] & {$[3.6], J-[3.6]$} & 741 & 14 & \\
C-rich AGB & {$[3.6], J-[3.6]$} & 264 & 5 & Red \\
O-rich AGB & {$[3.6], J-[3.6]$} & 477 & 8 & Blue \\
x-AGB & {$[4.5], J-[4.5]$} & 21 & 0.4 & Green \\
Background galaxies & {$[4.5], J-[4.5]$} & 115 & 2 & Magen $\backslash$ rlapta \\
\hline
\end{tabular}

(i) $\mathrm{x}$-AGB stars (a combination of our selection and Boyer et al. 2015b) and LAVs without determined period that are expected to be LPVs near the end of the AGB phase (e.g. Schröder et al. 1999; Fraser et al. 2008; Soszyński et al. 2009).

(ii) RSGs that do not have a determined period but must be good candidates for being LPVs. We note that any meaningful period determination of RSGs may require decades of observations (e.g. Pierce, Jurcevic \& Crabtree 2000; Kiss, Szabó \& Bedding 2006).

Based on their location in the CMDs and with respect to isochrones (Fig. 2; Padova models from Marigo et al. 2017), our sample occupies the same region as the LPVs in our previous works.

The sample is summarized in Table 4; of the x-AGB stars, eight were identified as LPVs or LAVs, hence the number of unique sources is 53 (two of the RSGs are also LPVs but they have not been counted in that category in the table).

We limit the field of study to the smaller of the two, Boyer et al. (2015a) and Menzies et al. (2015), measuring $11.8 \mathrm{kpc}^{2}$ (after deprojection). Our sample is shown in Fig. 3, in relation to the general stellar distribution and the neutral hydrogen gas. As the half-light radius suggests, as many sources could be expected to be found (just) outside that radius as within that radius. While there is no direct confirmation of membership for any of these sources, their concentration on the (small portion of) sky strongly suggests that most - if not all - are associated with IC 1613.

\section{METHOD: DERIVING THE SFH}

The method we employ here was developed in Javadi et al. (2011, 2017). We limit ourselves to a brief description along with the model parametrizations determined for our case.

The SFH is the rate at which gas is converted into stars, $\xi$ (in $\mathrm{M}_{\odot} \mathrm{yr}^{-1}$ ), as a function of time. The amount of stellar mass, $\mathrm{d} M$, produced in a time interval $\mathrm{d} t$ is

$\mathrm{d} M(t)=\xi(t) \mathrm{d} t$.

We can find the number of formed stars from the total produced stellar mass by

$\mathrm{d} N(t)=\frac{\int_{\min }^{\max } f_{\mathrm{IMF}}(m) \mathrm{d} m}{\int_{\min }^{\max } f_{\mathrm{IMF}}(m) m \mathrm{~d} m} \mathrm{~d} M(t)$,

where $f_{\mathrm{IMF}}$ is the initial mass function (IMF). We use the IMF defined in Kroupa (2001):

$f_{\mathrm{IMF}}=A m^{-\alpha}$,

where $A$ is the normalization coefficient and $\alpha$ is a factor that depends on the mass range:

$\alpha=\left\{\begin{array}{l}+0.3 \pm 0.7 \text { for } \min <\frac{m}{\mathrm{M}_{\odot}}<0.08, \\ +1.3 \pm 0.5 \text { for } 0.08<\frac{m}{\mathrm{M}_{\odot}}<0.5, \\ +2.3 \pm 0.3 \text { for } 0.5<\frac{m}{\mathrm{M}_{\odot}}<\max .\end{array}\right.$

The minimum and maximum mass in Kroupa's IMF were assumed to be 0.02 and $200 \mathrm{M}_{\odot}$, respectively.

Given that we use LPVs as proxies for the underlying stellar populations, we need to relate the number of variable stars to the total number of stars, $N$, that were formed in $\mathrm{d} t$. We note that in this section 'LPVs' stand for all identified LPVs and other candidates that we expect to be in this phase (cf. Section 2.4). If stars with mass between $m(t)$ and $m(t+\mathrm{d} t)(t$ is lookback time) are now LPVs, then the number of LPVs created between times $t$ and $t+\mathrm{d} t$ is

$\mathrm{d} n(t)=\frac{\int_{m(t)}^{m(t+\mathrm{d} t)} f_{\mathrm{IMF}}(m) \mathrm{d} m}{\int_{\min }^{\max } f_{\mathrm{IMF}}(m) \mathrm{d} m} \mathrm{~d} N(t)$. 

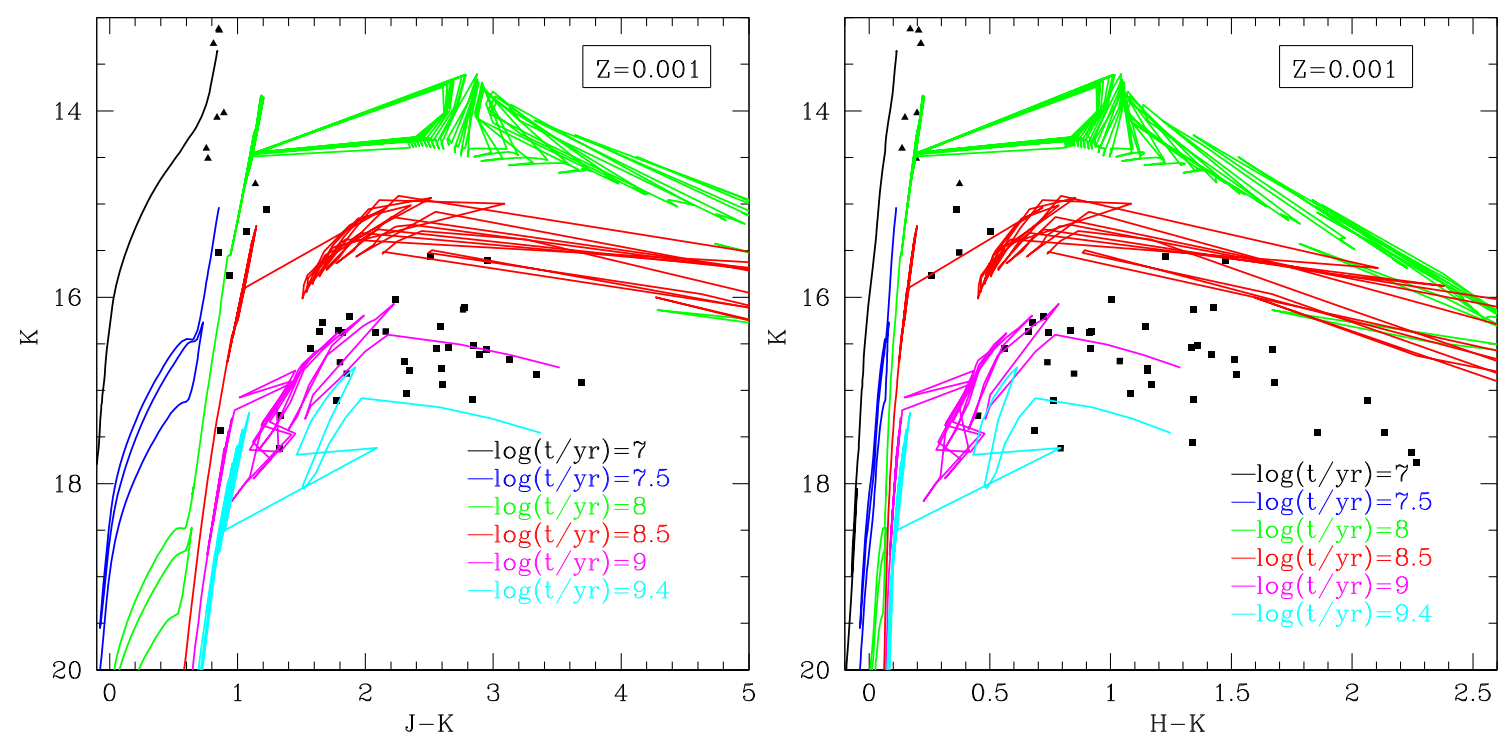

Figure 2. $K$ versus $J-K$ (left) and $H-K$ (right) with our selection of evolved stars (squares: AGB LPVs; triangles: RSGs), overlain with isochrones from Padova models (Marigo et al. 2017) labelled with logarithmic ages.

Table 4. Description of our sample to derive the SFH in IC 1613 (total excludes duplicates).

\begin{tabular}{lcc}
\hline Population & $N$ & Reference \\
\hline LPV & 14 & Menzies et al. (2015) \\
LAV & 9 & Menzies et al. (2015) \\
x-AGB & 30 & Boyer et al. (2015b) (cf. Section 2.3) \\
RSG & 8 & Menzies et al. (2015) \\
Total & 53 & \\
\hline
\end{tabular}

By substituting equations (1) and (2) into equation (5) we have

$\mathrm{d} n(t)=\frac{\int_{m(t)}^{m(t+\mathrm{d} t)} f_{\mathrm{IMF}}(m) \mathrm{d} m}{\int_{\min }^{\max } f_{\mathrm{IMF}}(m) m \mathrm{~d} m} \xi(t) \mathrm{d} t$.

The number of LPVs, $\mathrm{d} n$ ', we can observe in an age bin, $\mathrm{d} t$, depends on the size of the bin $(\mathrm{d} t)$ and on the duration of the LPV stage $(\delta t)$ :

$\mathrm{d} n^{\prime}(t)=\frac{\delta t}{\mathrm{~d} t} \mathrm{~d} n(t)$

Finally, by combining the above equations, we obtain a relation to give the SFR based on LPV counts:

$\xi(t)=\frac{\int_{\min }^{\max } f_{\mathrm{IMF}}(m) m \mathrm{~d} m}{\int_{m(t)}^{m(t+\mathrm{d} t)} f_{\mathrm{IMF}}(m) \mathrm{d} m} \frac{\mathrm{d} n^{\prime}(t)}{\delta t}$.

In order to relate a LPV's brightness to its mass, and its mass to its age (lookback time $t$ ) we appeal to stellar evolution models. The most suitable models are those from the Padova group, as argued extensively in Javadi et al. (2011, 2017), Rezaeikh et al. (2014), and Golshan et al. (2017). Here we use the latest version (PARSEC v1.2S + COLIBRI PR16; Marigo et al. 2017), which was improved upon the previous one (Marigo et al. 2008) resulting in a better estimation of the birth mass and pulsation time-scale. Some cases of improvements that matter for us here are: new TP-AGB evolutionary tracks and atmosphere models for O-rich and C-rich stars; the complete thermal pulse cycles, with a full description of the in-cycle changes in the stellar parameters; new pulsation models to describe the fundamental and first overtone modes of LPVs; and new dust models that consider the growth of the grains during the AGB evolution. That said, the evolutionary tracks of the youngest (most massive) stars $\left(\log M / \mathrm{M}_{\odot} \gtrsim 0.7\right)$ were not updated and still hail from the 2008 publication.

The LPVs are assumed to have reached their maximum near-IR brightness. Hence we apply a mass $-K$-band magnitude relation appropriate for the metallicity and distance modulus of IC 1613 ( $Z=$ 0.001 and $\mu=24.37$ mag; left-hand panel of Fig. 4; all the figures and tables for other metallicities we use in this paper are presented in Appendix A). While the $K$-band magnitude varies during the radial pulsation cycle, the photometry we are using are the mean magnitudes over several epochs and thus representative of the luminosity associated with the nuclear burning inside the star. The evolution of super-AGB stars toward the brighter $K$-band magnitudes has been omitted from the models, thus we interpolate the models over that range in mass (see Javadi et al. 2011 for details). The coefficients of the linear fitting between $K$-band magnitude and mass are listed in Table 5.

The onset of dust formation reddens and dims the stars, and we therefore need to apply a correction to bring them back to their photospheric peak brightness level. Because the evolutionary time-scale shortens dramatically once dust formation sets in, the same brightness level found in the model at the end point of stellar evolution corresponds to the nuclear burning luminosity that can be directly related to the birth mass. Because some of the stars in our sample do not have $J$-band detections, we use $H-K$ colours to determine this correction. As clearly discernible in Fig. 2, the reddened parts of the tracks/isochrones are bimodal in terms of their slope, based on whether the dust is O-rich or C-rich (Albert, Demers \& Kunkel 2000; Menzies et al. 2015). Therefore, we apply the following correction:

$K_{\text {cor }}=K+a_{\text {oxygen }}\left[(H-K)-(H-K)_{0}\right]$

for O-rich stars. For C-rich stars according to the isochrones in the right-hand panels of Fig. 2, we have

$K_{\text {cor }}=K+a_{\text {carbon }}\left[(H-K)-(H-K)_{0}\right]$,

where $a_{\text {oxygen }}=-1.2$ and $a_{\text {carbon }}=-0.85$ are the average slopes of the isochrone after the peak in the $K$ versus $H-K$ CMDs, and 


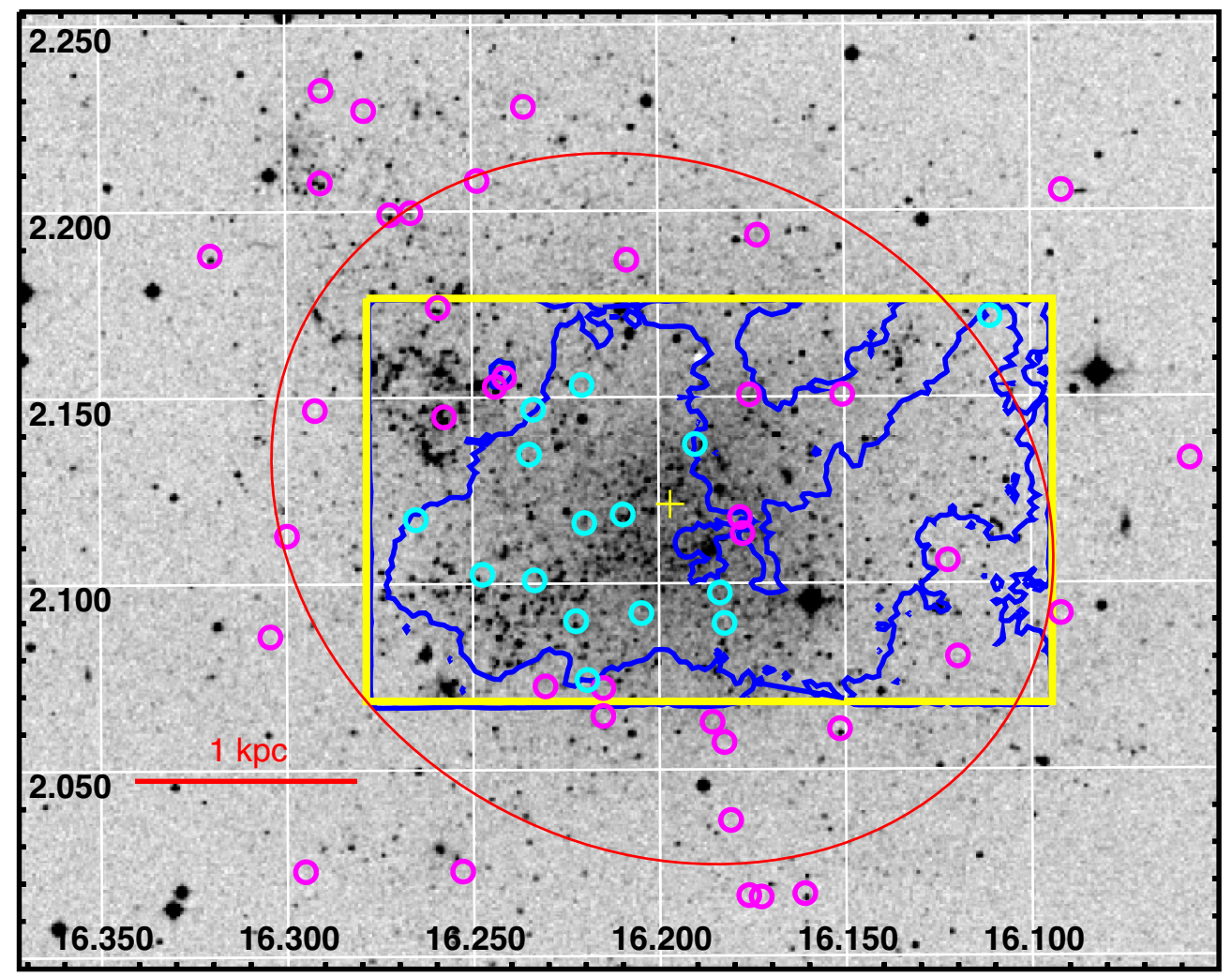

Figure 3. Spatial distribution of our stellar sample (magenta and cyan circles) and neutral hydrogen shells (Hi; the largest blue contours in the yellow box). The stars of the sample that are inside the approximate boundary of the main H I supershell (Silich et al. 2006) are shown with cyan circles. The yellow cross indicates the optical centre of IC 1613, with the red ellipse outlining the half-light radius $\left(r_{\mathrm{h}}=6.5\right.$ arcmin, position angle PA $\left.=58^{\circ}\right)$. The $($ archival) optical image (at $468 \mathrm{~nm}$ wavelength) in the background was made with the United Kingdom Schmidt Telescope.
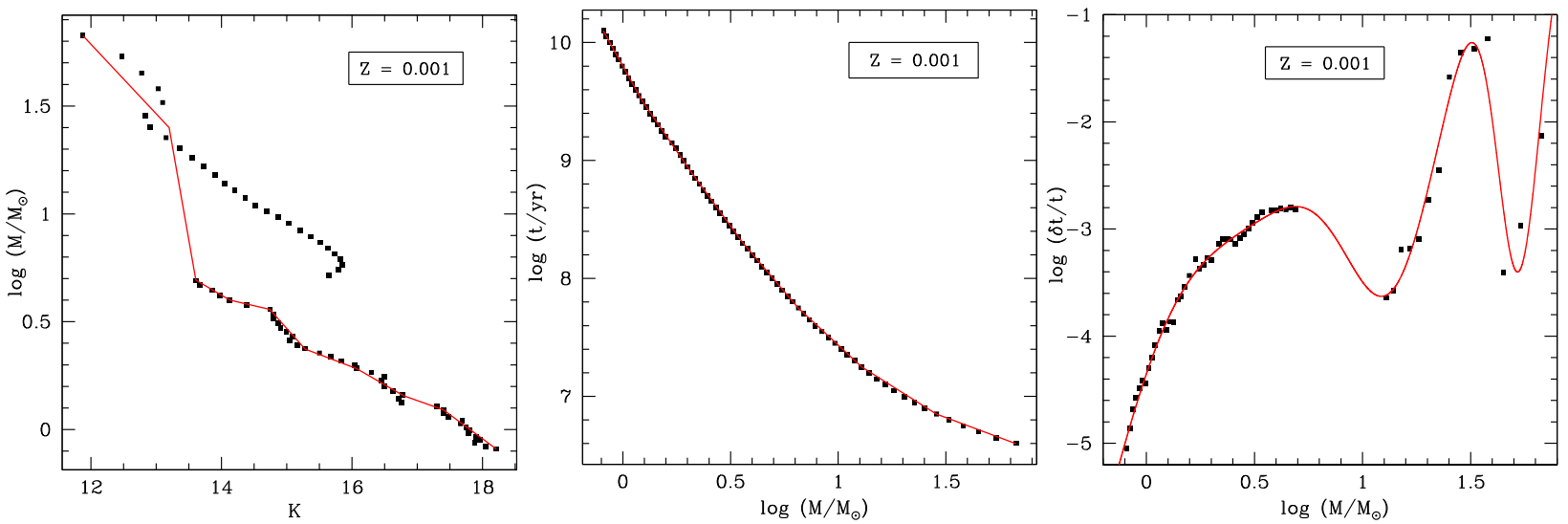

Figure 4. Left: relation between birth mass and $K$-band magnitude at the end point of stellar evolution for $Z=0.001$ and a distance modulus of $\mu=24.37$ mag. Solid lines are fits, in which the function is interpolated over the super-AGB phase $\left(0.7 \lesssim \log M / \mathrm{M}_{\odot} \lesssim 1.3\right)$. Middle: same as the left, but for the relation between age (at the end point of stellar evolution) and birth mass. Right: same as the left, but for the relation between relative pulsation duration and birth mass. The points show the ratio of pulsation duration to age versus mass; the solid lines are multiple-Gaussian fits, also interpolated over the super-AGB regime.

the average colours at the peak brightness are $(H-K)_{0}=1.05 \mathrm{mag}$ for O-rich stars and $(H-K)_{0}=0.8$ mag for C-rich stars. In some cases there is spectroscopic evidence for the chemical class of the object (Albert et al. 2000; Menzies et al. 2015); where this is not the case we have relied on photometric classification criteria (Boyer et al. 2015b; Menzies et al. 2015; Sibbons et al. 2015). The same selection criteria are also used for classification of $\mathrm{x}$-AGB stars.
The relation between mass and age for LPVs is presented in the middle panel of Fig. 4, with the coefficients of linear fits listed in Table 6 , for a metallicity of $Z=0.001$.

We derived the relative pulsation duration (ratio of pulsation time-scale to age) for a given birth mass from the Padova models; these relations are presented in the right-hand panel of Fig. 4, and parametrized by a set of four Gaussian functions listed in Table 7, 
Table 5. Fitted equations for the relation between birth mass and $K$-band magnitude, $\log M / \mathrm{M}_{\odot}=a K+b$, for a distance modulus of $\mu=24.37$ mag.

\begin{tabular}{lcc}
\hline $\begin{array}{l}Z=0.001 \\
a\end{array}$ & $b$ & Validity range \\
\hline$-0.322 \pm 0.009$ & $5.560 \pm 0.168$ & $K \leq 13.20$ \\
$-1.726 \pm 0.051$ & $24.19 \pm 0.720$ & $13.20<K \leq 13.61$ \\
$-0.176 \pm 0.005$ & $3.092 \pm 0.009$ & $13.61<K \leq 14.13$ \\
$-0.072 \pm 0.002$ & $1.617 \pm 0.048$ & $14.13<K \leq 14.74$ \\
$-0.339 \pm 0.016$ & $5.552 \pm 0.168$ & $14.74<K \leq 15.28$ \\
$-0.116 \pm 0.004$ & $2.140 \pm 0.063$ & $15.28<K \leq 16.07$ \\
$-0.175 \pm 0.006$ & $3.090 \pm 0.009$ & $16.07<K \leq 16.79$ \\
$-0.107 \pm 0.003$ & $1.944 \pm 0.057$ & $16.79<K \leq 17.40$ \\
$-0.225 \pm 0.006$ & $4.008 \pm 0.120$ & $K>17.40$ \\
\hline & &
\end{tabular}

Table 6. Fits to the relation between age and birth mass, $\log t=a \log M / \mathrm{M}_{\odot}$ $+b$.

\begin{tabular}{lcc}
\hline $\begin{array}{l}Z=0.001 \\
a\end{array}$ & $b$ & Validity range \\
\hline$-3.289 \pm 0.099$ & $9.801 \pm 0.294$ & $\log M / \mathrm{M}_{\odot} \leq 0.10$ \\
$-2.649 \pm 0.078$ & $9.743 \pm 0.291$ & $0.10<\log M / \mathrm{M}_{\odot} \leq 0.31$ \\
$-2.510 \pm 0.075$ & $9.698 \pm 0.291$ & $0.31<\log M / \mathrm{M}_{\odot} \leq 0.55$ \\
$-2.132 \pm 0.063$ & $9.487 \pm 0.258$ & $0.55<\log M / \mathrm{M}_{\odot} \leq 0.81$ \\
$-1.701 \pm 0.051$ & $9.137 \pm 0.273$ & $0.81<\log M / \mathrm{M}_{\odot} \leq 1.11$ \\
$-1.153 \pm 0.036$ & $8.528 \pm 0.255$ & $1.11<\log M / \mathrm{M}_{\odot} \leq 1.45$ \\
$-0.678 \pm 0.020$ & $7.836 \pm 0.234$ & $\log M / \mathrm{M}_{\odot}>1.45$ \\
\hline
\end{tabular}

Table 7. Fits to the relation between relative pulsation duration $(\delta t / t$, where $t$ is the age and $\delta t$ is the pulsation duration) and birth mass, $\log (\delta t / t)=$ $\Sigma_{i=1}^{4} a_{i} \exp \left[-\left(\log M / \mathrm{M}_{\odot}-b_{i}\right)^{2} / c_{i}^{2}\right]$.

\begin{tabular}{lccc}
\hline $\begin{array}{l}Z=0.001 \\
i\end{array}$ & $a$ & $b$ & $c$ \\
\hline 1 & -53.46 & -0.115 & 0.749 \\
2 & -02.62 & +1.152 & 0.316 \\
3 & +48.48 & -0.078 & 0.697 \\
4 & -03.23 & +1.722 & 0.139 \\
\hline
\end{tabular}

for a metallicity of $Z=0.001$ (relations for other metallicities used in this paper can be found in Appendix A).

To calculate the SFH, we thus employ the following procedure.

(i) Correct the $K$-band magnitude for dust attenuation.

(ii) Use the corrected $K$-band magnitude and the mass $-K$-band equation (Table 5 ) to obtain the birth mass.

(iii) Use the birth mass and the age-mass equation (Table 6) to obtain the age.

(iv) Use the birth mass and the pulsation duration-mass equation (Table 7) to obtain the pulsation time-scale.

(v) Choose appropriate age bins and apply equation (8) to calculate the SFR in each of those bins.

For each bin, a statistical error can be derived from Poisson statistics as follows:

$\sigma_{\xi(t)}=\frac{\sqrt{N}}{N} \xi(t)$

where $N$ is the number of stars in each age bin.

\section{RESULTS}

Using our sample of evolved stars (Section 2.4) and applying our method (Section 3), we estimate SFRs in IC 1613 over the broad time interval from $30 \mathrm{Myr}$ to $\sim 5 \mathrm{Gyr}$ ago. The observational reasons for being unable to determine SFRs at earlier epochs will be described below. Because the metallicity is expected to increase over time as a result of the chemical evolution driven by nucleosynthesis and mass loss, we first apply our method assuming different metallicities and then re-analyse it adopting instead the linear agemetallicity relation (AMR) from Skillman et al. (2014) for values $4 \times 10^{-4}<Z<4 \times 10^{-3}$ (corresponding to 13 Gyr ago $<t<$ now)

We start by examining the recent $\mathrm{SFH}$ at a constant metallicity. The SFR as a function of lookback time in the last Gyr is shown in the left-hand panel of Fig. 5. For this epoch we assumed $Z=0.003$. The horizontal bars represent the spread in age within each bin, while the vertical bars represent the statistical errors. The mean SFR over the last $200 \mathrm{Myr}(\log t(\mathrm{yr}) \sim 8.3)$ in the central $11.8 \mathrm{kpc}^{2}$ is $\xi \sim$ $(0.5 \pm 0.2) \times 10^{-3} \mathrm{M}_{\odot} \mathrm{yr}^{-1} \mathrm{kpc}^{-2}$; it is marginally higher in the central $2.2 \mathrm{kpc}^{2}, \xi \sim(0.65 \pm 0.3) \times 10^{-3} \mathrm{M}_{\odot} \mathrm{yr}^{-1} \mathrm{kpc}^{-2}$. This agrees well with the result derived by Bernard et al. (2007): they found a mean SFR of $\xi \sim(1.6 \pm 0.8) \times 10^{-3} \mathrm{M}_{\odot} \mathrm{yr}^{-1} \mathrm{kpc}^{-2}$ in the central part $(r \leq 2.5 \mathrm{arcmin})$ of the galaxy for the last $300 \mathrm{Myr}(\log t(\mathrm{yr}) \sim$ 8.5), replicating the result obtained by Cole et al. (1999) for a central $\sim 0.22 \mathrm{kpc}^{2}$ region on the basis of the main-sequence luminosity distribution. Cole et al. also found that the SFR was $\sim 50$ per cent higher 400-900 Myr ago $(8.6<\log t(\mathrm{yr})<8.9)$. While based on just 19 stars in the central $2.2 \mathrm{kpc}^{2}$, we find $\xi \sim(0.9 \pm 0.3) \times$ $10^{-3} \mathrm{M}_{\odot} \mathrm{yr}^{-1} \mathrm{kpc}^{-2} 500-900 \mathrm{Myr}$ ago (Fig. 5, right), i.e. a very similar SFH as that determined by Cole et al.

Subsequently, we applied this process for all epochs and five metallicities $(Z=0.0004,0.0007,0.001,0.002$, and 0.003$)$; the result is shown in Fig. 6. Clearly, the result is sensitive to the adopted metallicity, so in order to trace back the SFH we need to take into account the variation of metallicity with lookback time.

Skillman et al. (2014) modelled the CMDs in a small field near the half-light radius to determine the SFH for the full $13 \mathrm{Gyr}$ of evolution. They found that most - if not all - stars in IC 1613 formed after the epoch of reionization $(\sim 12.8 \mathrm{Gyr}$ ago $)$, without a dominant formation epoch. To compare with their result, we adopt a similar AMR as they did: $Z=0.003$ for the last Gyr $(\log t(\mathrm{yr})$ $<9), Z=0.002$ for $1<t<2 \mathrm{Gyr}(9<\log t(\mathrm{yr})<9.3)$, and $Z=$ 0.0007 for $t>2 \mathrm{Gyr}(\log t(\mathrm{yr})>9.3)$. We thus find a mean value of the SFR across IC 1613 over the last Gyr of $\xi=(3.0 \pm 0.5) \times$ $10^{-4} \mathrm{M}_{\odot} \mathrm{yr}^{-1} \mathrm{kpc}^{-2}$ (Fig. 7), in excellent agreement with Skillman et al., who found $\xi \sim 3.4 \times 10^{-4} \mathrm{M}_{\odot} \mathrm{yr}^{-1} \mathrm{kpc}^{-2}$.

However, Skillman et al. (2014) found SFRs at earlier epochs (1 $<t<2$ Gyr and $2<t<5$ Gyr) that are two to three times higher than our results, and we could not trace star formation beyond $\sim 5 \mathrm{Gyr}$ ago. We now discuss how the SFH we derive for lookback times $t$ $>9$ Gyr may have been biased.

The truncated SFH (beyond 5 Gyr) is mainly due to observational limitations with regard to our method.

(i) The sample of LPVs comes from the survey by Menzies et al. (2015), which quotes a completeness limit of $K_{\mathrm{s}} \approx 18 \mathrm{mag}$ and will have missed red variables with $\left(H-K_{\mathrm{s}}\right)>2.0 \mathrm{mag}$ and $K_{\mathrm{s}}$ $>16.3 \mathrm{mag}$. In order to trace the SFH to $t>5 \mathrm{Gyr}(\log t(\mathrm{yr})>$ 9.7) at low metallicity $(Z \sim 0.0007)$, however, we need stars that have dereddened $K$-band magnitudes fainter than $\sim 18$ mag (see the isochrone diagram in Fig. A1).

(ii) Regarding x-AGB stars, the Spitzer sample from Boyer et al. (2015a) is not flux limited, and the near-IR data from Sibbons et al. 

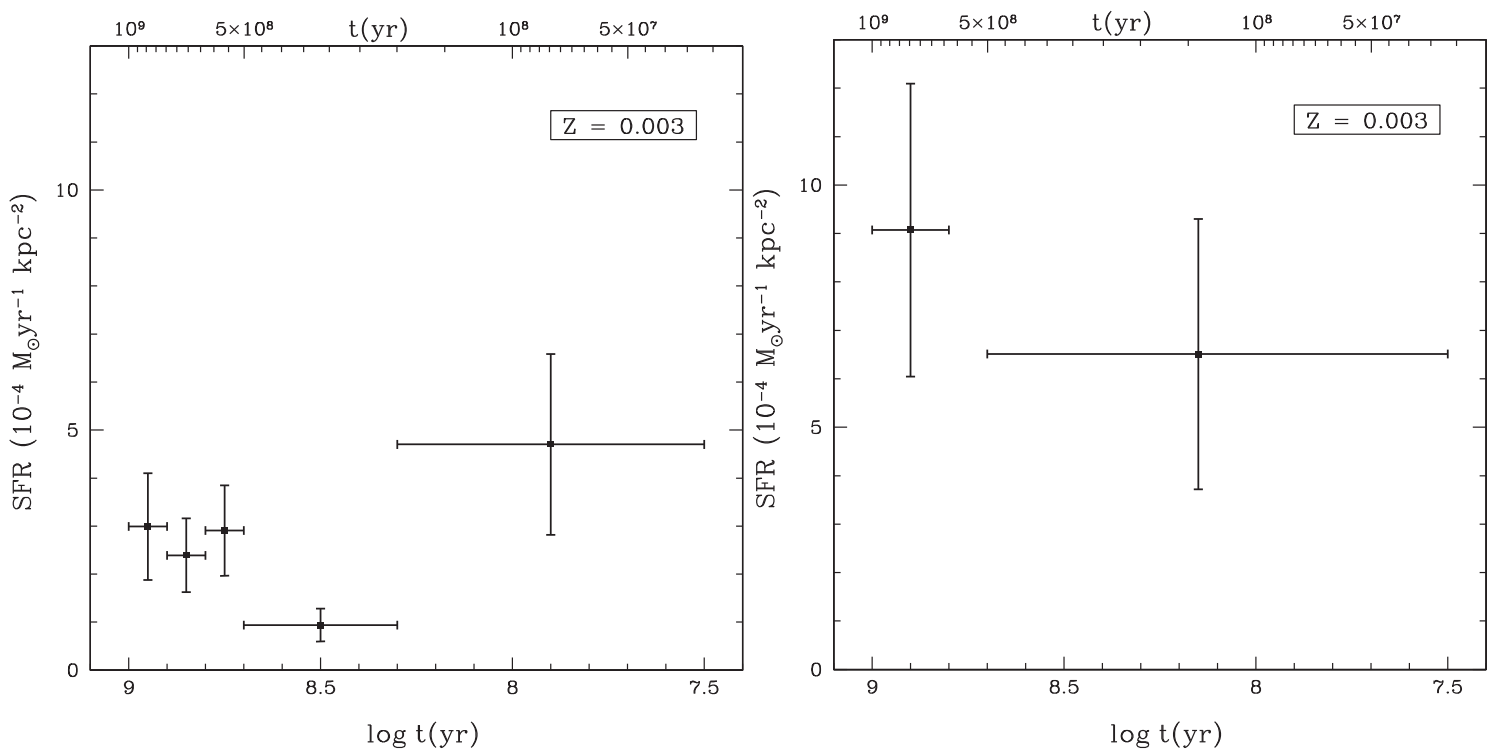

Figure 5. Left: recent SFH (last Gyr) in a $\sim 200 \operatorname{arcmin}^{2}\left(\sim 11.8 \mathrm{kpc}^{2}\right)$ of IC 1613 assuming a constant metallicity of $Z=0.003$. Right: same as left, but in $\sim 36 \operatorname{arcmin}^{2}\left(\sim 2.2 \mathrm{kpc}^{2}\right)$ in the central part of the galaxy.

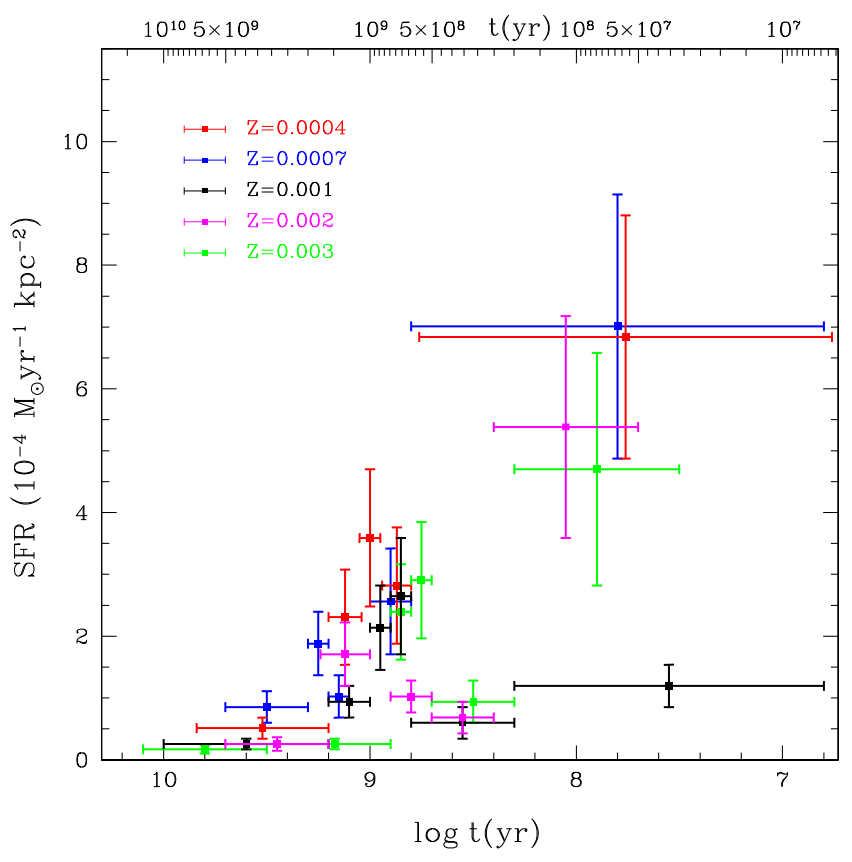

Figure 6. IC $1613 \mathrm{SFHs}$ for different metallicities.

(2015) are deep enough $(J<19.7, H<19.3$, and $K<18.9$ mag) to provide counterparts to place them in the CMD. However, most $\mathrm{x}$-AGB stars are carbon stars, with the odd more massive OH/IR star - cf. Woods et al. (2011), and carbon stars arise from stars born not longer than $~ 5.5 \mathrm{Gyr}$ ago (Dell Agli et al. 2016). Thus, these $\mathrm{x}$-AGB samples do not make up for the incompleteness of the variability survey by Menzies et al. beyond $5 \mathrm{Gyr}$.

The reasons for the discrepancy between our SFR determinations a few Gyr ago and those by Skillman et al. (2014) are more subtle, and probably include the following.

(i) It is likely that the completeness limits of the monitoring survey and possibly the near-IR complement to the Spitzer survey

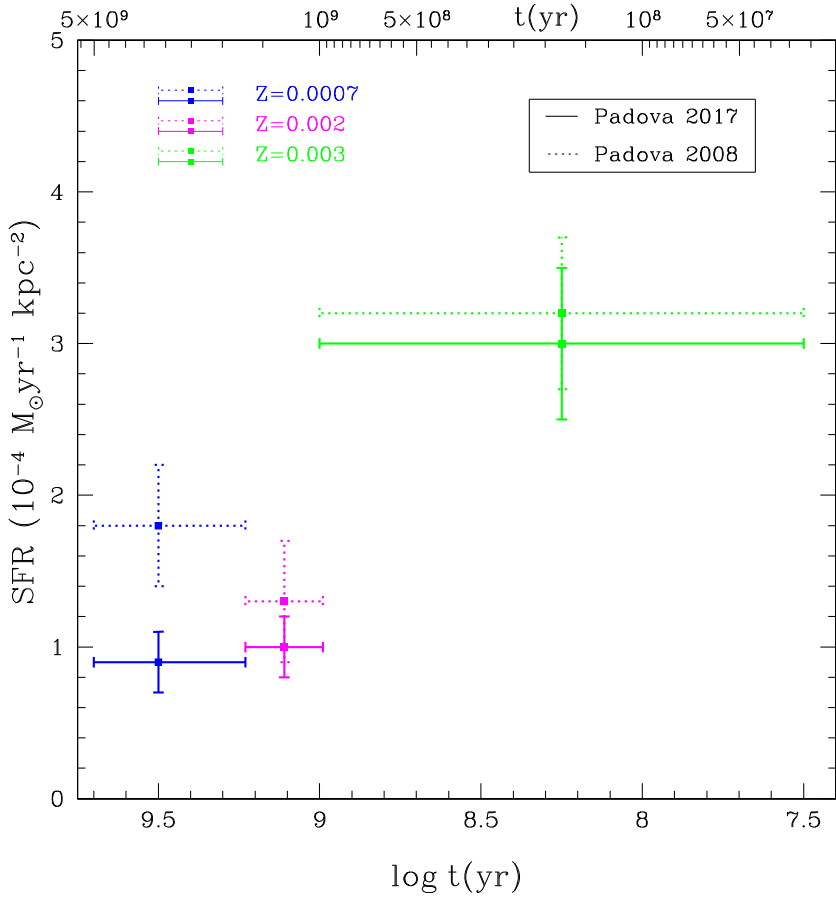

Figure 7. The SFH in IC 1613 with the adoption of an AMR akin that derived by Skillman et al. (2014), using the pulsation durations from Marigo et al. (2017) (solid) and Marigo et al. (2008) (dotted).

start to deplete our sample of sources at multi-Gyr ages. This is not an issue for the most recent Gyr, which is confirmed by the good correspondence with the results in the literature as described above.

(ii) While the field studied by Skillman et al. (2014) may be representative of the bulk of IC 1613 in recent times, this may not be true at earlier epochs. We tried to find the SFR on the same location as their field (RA $\sim 1^{\mathrm{h}} 4^{\mathrm{m}} 30^{\mathrm{s}}$ and Dec. $\sim 2^{\circ} 9^{\prime}$ ), but the sample became limited to just one star and this did not yield a meaningful SFR. 

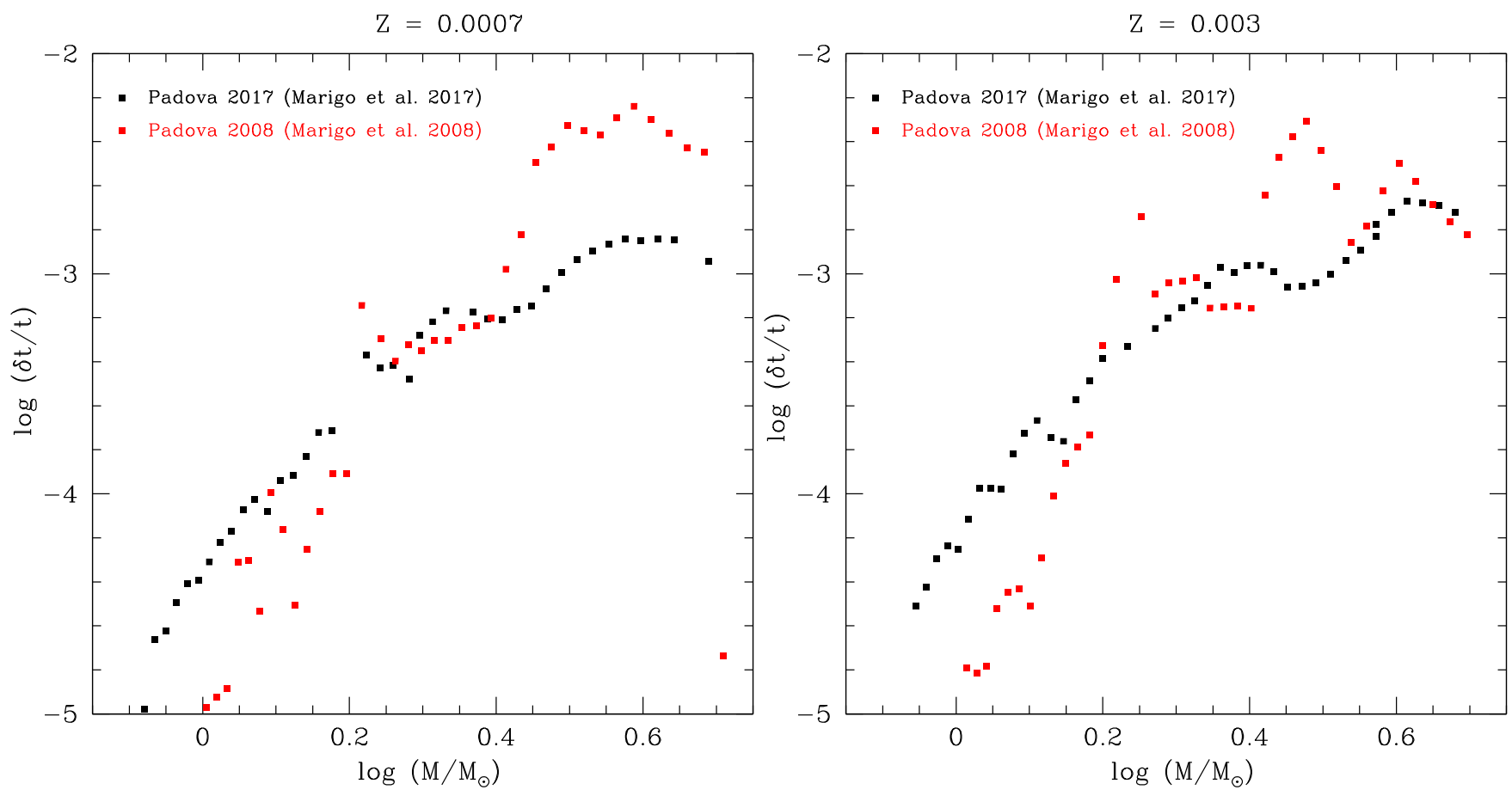

Figure 8. The differences between the pulsation durations $(\delta t)$ from the most recent Padova models (Marigo et al. 2017, black) and previous version (Marigo et al. 2008, red) are $Z=0.0007$ (left) and $Z=0.003$ (right).

(iii) The pulsation durations $(\delta t)$ may have been overestimated, which would immediately result in an underestimated SFR. Fig. 8 shows the differences between the Marigo et al. (2017) and Marigo et al. (2008) pulsation durations. For stars with $0.3 \lesssim \log M / \mathrm{M}_{\odot} \lesssim$ 0.7 the pulsation duration was shortened, while for intermediateand low-mass stars with $\log M / \mathrm{M}_{\odot} \lesssim 0.3$ the duration was lengthened. Our results are in better agreement with the results from Skillman et al. (2014) when using the 2008 models (dotted lines in Fig. 7). For $t>2$ Gyr $(\log t(\mathrm{yr})>9.3)$ the SFR using the Marigo et al. (2008) pulsation durations doubles and becomes $\sim 60$ per cent of the SFR derived by Skillman et al. $\left(\xi \sim 3 \times 10^{-4} \mathrm{M}_{\odot} \mathrm{yr}^{-1} \mathrm{kpc}^{-2}\right)$; for $1<t<2 \operatorname{Gyr}(9<\log t(\mathrm{yr})<9.3)$ the SFR would also increase but only to $\sim 30$ percent of the SFR derived by Skillman et al. ( $\xi$ $\sim 3.6 \times 10^{-4} \mathrm{M}_{\odot} \mathrm{yr}^{-1} \mathrm{kpc}^{-2}$ ). This is not the first time that results suggest that the pulsation durations in the Padova models may be too generous: Javadi et al. (2017) (see also Javadi et al. 2013) found corrections of a factor of $\sim 3$ were needed at solar and slightly subsolar metallicity. It appears that the 2008 Padova models are preferred over the 2017 models, at least for older ages at low metallicities, but still need to be corrected by a factor of $\sim 2-3$.

In any case we do not find a discrete epoch of enhanced (or suppressed) star formation (Fig. 7) that could be linked to external triggering mechanisms. This supports the notion that IC 1613 has evolved in isolation for at least the past $5 \mathrm{Gyr}$ (Cole et al. 1999; Stinson et al. 2007; Skillman et al. 2014).

\section{DISCUSSION}

\subsection{Galactocentric radial gradient of the SFH}

Galactocentric radial gradients are imprinted with the dynamical history and propagation of star formation. It appears that stellar population gradients are universal in dwarf galaxies, in the sense
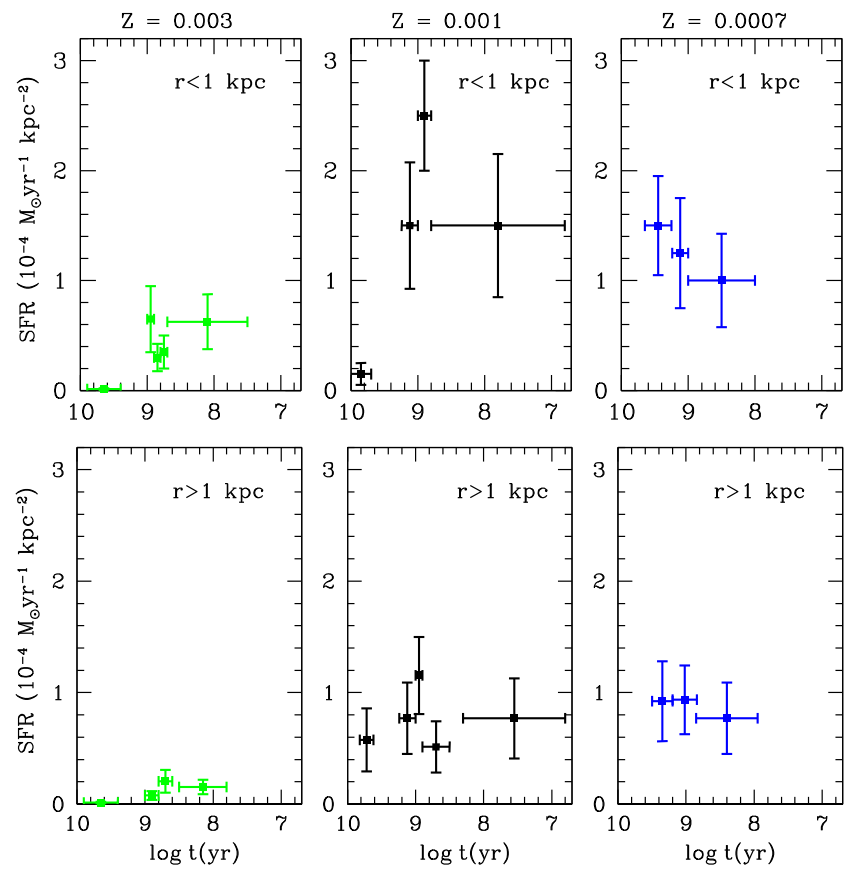

Figure 9. SFHs of two regions at different galactocentric radii $(r<1 \mathrm{kpc}$ and $r>1 \mathrm{kpc}$ ) for different metallicities.

that the mean age of the stellar population is younger towards the centre of the galaxy (e.g. Hidalgo et al. 2013; Skillman et al. 2014).

To examine radial gradients in IC 1613, we divided our sample into two parts - an inner $(r<1 \mathrm{kpc})$ and an outer $(r>$ $1 \mathrm{kpc})$ region. The resulting SFHs are shown in Fig. 9. In the inner part, the mean SFR over the past Gyr $(Z \sim 0.003)$ was $\xi \sim 0.7 \times 10^{-4} \mathrm{M}_{\odot} \mathrm{yr}^{-1} \mathrm{kpc}^{-2}$, in the outer part it was $\xi \sim$ 
$0.2 \times 10^{-4} \mathrm{M}_{\odot} \mathrm{yr}^{-1} \mathrm{kpc}^{-2}$. Clearly, the inner part contains more young stars relative to the outer region, but this is in part due to the radially decreasing overall stellar density. However, when considering the SFRs at older times and lower metallicity, they increase more rapidly in the outer parts than in the inner parts of IC 1613. In fact, at the lower metallicities $(Z \sim 0.0007)$ the SFR in the outer galaxy rivals that near the centre.

Another way of quantifying this gradient is in terms of the ratio of SFRs at older times $(\log t(\mathrm{yr})>9.2, Z=0.0007)$ to those at recent times $(\log t(\mathrm{yr})<9, Z=0.003)$. In the inner region this ratio is $\sim 2$, while in the outer region it is $\sim 7$. Considering the error bars (minimum of old SFR and maximum of recent SFR) decrease the ratio to $\sim 1$ for the inner part and $\sim 3$ for the outer part. This outside-in evolution scenario (at least for the most recent $\sim 5 \mathrm{Gyr}$ ) is in agreement with what is typically found in dwarf galaxies (e.g. Hidalgo, Marín-Franch \& Aparicio 2003; Hidalgo, Aparicio \& Gallart 2008; Hidalgo et al. 2013) and differs from what is found in the low-mass spiral galaxy M33, for instance (Javadi et al. 2017).

As we described in the result section, because of incompleteness of the surveys it is possible that we have underestimated the SFR older than a Gyr. Likewise, the discrepant pulsation durations between the 2008 and 2017 Padova models could have affected the older SFRs. But this is true for both the inner and outer part in this section, and we do not expect the observed gradient to change. Furthermore, the SFH over the last Gyr in the central part (right-hand panel of Fig. 5) compared to that in our larger field of view (left-hand panel of Fig. 5) shows an obvious concentration of recent star formation in the central part of the galaxy.

\subsection{The origin of the main hydrogen supershell}

The neutral ISM within dwarf galaxies often shows holes, arcs, and shells. These structures are typically explained by the (combined) effects of stellar winds from massive stars and supernova ( $\mathrm{SN}$ ) explosions (Dyson \& de Vries 1972; Weaver et al. 1977), but this scenario struggles to explain the largest structures (Silich et al. 2006 and references therein).

With regard to IC 1613, Silich et al. (2006) identified several H I supershells, including 'main supershell' - an H I hole of $1 \mathrm{kpc}$ in diameter and surrounded by an $\mathrm{H}$ I ring centred at RA $=1^{\mathrm{h}} 4^{\mathrm{m}} 52^{\mathrm{s}}$ (16.2167), Dec. $=2^{\circ} 7^{\prime}(2.1167)$ (see Fig. 3). The HI mass of this supershell is $2.8 \times 10^{7} \mathrm{M}_{\odot}$. The absence of regular expansion and the thickness of the ring led Silich et al. to believe the shell has stalled. The wide range in age of the $\mathrm{OB}$ associations - up to $30 \mathrm{Myr}$ (Borissova et al. 2004) - found inside the shell, suggested to them that the shell was formed over a period of $\sim 30$ Myr.

Silich et al. (2006) simulated the formation of the main supershell for two different (thin and thick) galactic disc models, in both cases concluding a formation time-scale of $30 \mathrm{Myr}$. They thus estimated a mid-plane gas number density $n_{0} \sim 2.8(1.4) \mathrm{cm}^{-3}$ and a SFR of $\xi$ $\sim 7.5(2.3) \times 10^{-3} \mathrm{M}_{\odot} \mathrm{yr}^{-1}$ for their thin (thick) disc models. This is an order of magnitude larger than the in situ SFR they derived from $\mathrm{H} \alpha$ data, $\xi \sim(3-4) \times 10^{-4} \mathrm{M}_{\odot} \mathrm{yr}^{-1}$, leading them to reject the multiple $\mathrm{SN}$ scenario.

To revisit the SFR within the main supershell, we selected stars - 15 in total - from our sample that reside inside the contour outlining the main supershell in Silich et al. (2006) (Fig. 3). Assuming a constant metallicity of $Z=0.003$, we thus derive the

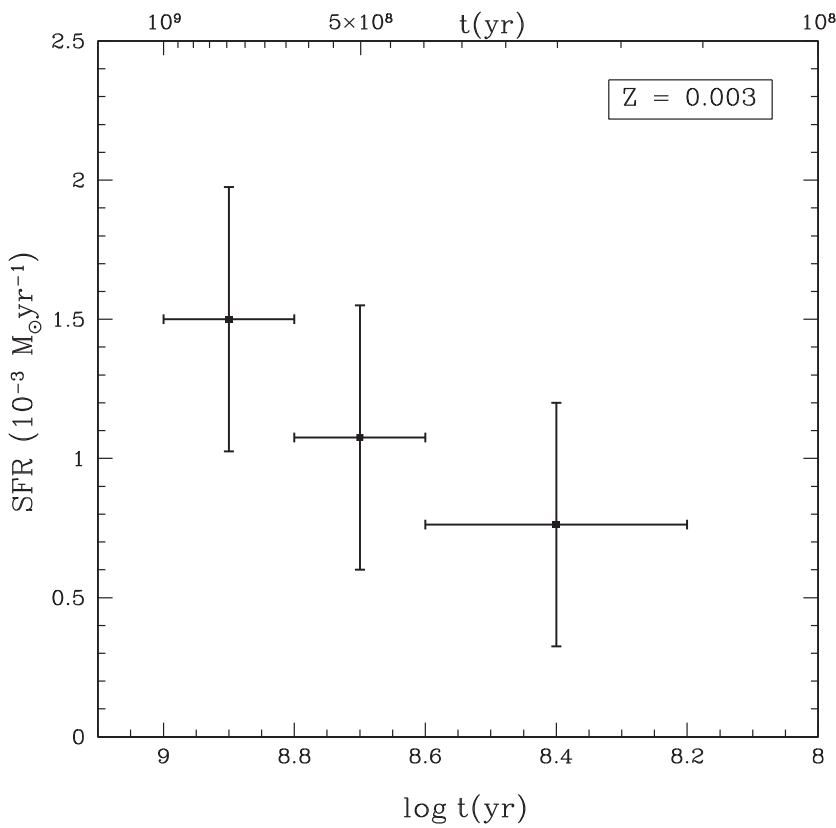

Figure 10. SFHs within the main supershell (Silich et al. 2006) of IC 1613 assuming a constant metallicity of $Z=0.003$.

recent SFH within the shell (Fig. 10). Because of the small number statistics we lack supergiants within this limited sample and thus cannot ascertain the SFR at $t<100 \mathrm{Myr}$. However, the SFR seems to have slowly but steadily decreased in recent times, and if this trend continued into the most recent $30 \mathrm{Myr}$, we would estimate a SFR that is compatible with the one derived from $\mathrm{H} \alpha$ by Silich et al. (2006). One may wonder, though whether the shell could in fact have formed over much longer time-scales, in excess of $100 \mathrm{Myr}$ - from which $\mathrm{OB}$ associations no longer exist.

\subsection{Ability of mass loss to sustain star formation}

Star formation depletes a galaxy of its gas, but stellar mass loss replenishes it. The question therefore is whether the latter can sustain the former.

Silich et al. (2006) derived a total HI mass in IC 1613 of $6.0 \times 10^{7} \mathrm{M}_{\odot}$, in good agreement with previous estimates from Lake \& Skillman (1989). Considering also the contribution from helium, the total mass of the ISM would be $\sim 9.0 \times 10^{7} \mathrm{M}_{\odot}$. Dell' Agli et al. (2016) used stellar evolution models to estimate the dust pro-

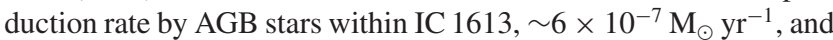
gas-to-dust mass ratio, $\sim 1000$, and hence a total mass return rate of $6 \times 10^{-4} \mathrm{M}_{\odot} \mathrm{yr}^{-1}$

The recent SFR of $\xi=(5.5 \pm 2) \times 10^{-3} \mathrm{M}_{\odot} \mathrm{yr}^{-1}$ we derived from the evolved star population (Fig. 5) exceeds the mass return rate by an order of magnitude. This means that the ISM will be depleted by star formation on a time-scale of $16.4 \mathrm{Gyr}$. Accounting for the mass return rate would stretch this by a small amount to $\sim 18.4$ Gyr. Considering the contribution of ionized mass of the galaxy (about a few times $10^{6}$ from Silich et al. 2006) can increase the estimation up to 10 per cent.

This assumes that star formation is 100 percent efficient, and that no ISM gas is blown out of the shallow gravitational potential well of the dwarf galaxy, so star formation at the current rate would unlikely continue for that long. On the other hand, the above estimate 
also neglects contributions to mass return by $\mathrm{SNe}$, luminous blue variables et cetera - though these do not make a huge difference when assessed over cosmological times (cf. Javadi et al. 2013). The expectation therefore is that IC 1613 cannot continue to form stars at the current rate for more than a few Gyr, unless gas is accreted from outside, for instance when (if) it traverses the halo from M31 or the Milky Way, or intergalactic gas if such reservoirs exist.

\section{SUMMARY OF CONCLUSIONS}

Selecting stars at the end points of their evolution, we applied a recently developed method to derive the SFH in the isolated Local Group dwarf irregular galaxy IC 1613. Our main findings are as follows.

(i) From a combination of near-/mid-IR CMDs we identified 21 $\mathrm{x}$-AGB stars, of which two stars had not been identified before.

(ii) We do not find any dominant period of star formation over the past 5 Gyr, which suggests that IC 1613 may have evolved in isolation for at least that long.

(iii) The SFH over the past few Gyr confirms those derived by other methods, with a radial gradient that indicates the mean age of the central population is younger than that in the outskirts.

(iv) The extrapolated rate of recent star formation within the main H I supershell falls short by an order of magnitude of the required recent SFR to have produced the shell.

(v) The current reservoir of interstellar gas may sustain star formation at the current rate for several Gyr into the future; however, the rate at which mass is returned will not extend that time for very much longer - indeed, star formation beyond the next few Gyr will diminish and eventually cease altogether unless gas can be accreted from outside.

\section{ACKNOWLEDGEMENTS}

We are deeply grateful to Sohrab Rahvar and Martha Boyer for their valuable comments and discussions. We thank Mehrdad Phoroutan Mehr for his kind comments. We would also like to thank Sergiy Silich, Tatyana Lozinskaya, and Alexei Moiseev for sharing their H I maps and their responses to our questions. Finally, The authors are indebted to the anonymous referee for the careful reading of the manuscript and for the helpful comments, which prompted them to improve the quality of this work.

\section{REFERENCES}

Albert L., Demers S., Kunkel W. E., 2000, AJ, 119, 2780

Bernard E. J., Aparicio A., Gallart C., Padilla-Torres C. P., Panniello M., 2007, AJ, 134, 1124

Blum R. D. et al., 2006, AJ, 132, 2034

Blumenthal G. R., Faber S. M., Primack J. R., Rees M. J., 1984, Nature, 311,517

Borissova J., Kurtev R., Georgiev L., Rosado M., 2004, A\&A, 413, 889

Boyer M. L., Skillman E. D., van Loon J. Th., Gehrz R. D., Woodward C. E., 2009, ApJ, 697, 1993

Boyer M. L. et al., 2015a, ApJS, 216, 10

Boyer M. L. et al., 2015b, ApJ, 800, 51
Britavskiy N. E., Bonanos A. Z., Mehner A., García-Álvarez D, Prieto J. L., Morrell N. I., 2014, A\&A, 562, A75

Carroll B. W., Ostlie D. A., 2007, An Introduction to Modern Astrophysics. Pearson Addison-Wesley, San Francisco

Chun S. H., Jung M., Kang M., Kim J. W., Sohn Y. J., 2015, A\&A, 578, A51

Cole A. A. et al., 1999, AJ, 118, 1657

Dekel A., Woo J., 2003, MNRAS, 344, 1131

Dell’Agli F., Di Criscienzo M., Boyer M. L., García-Hernández D. A., 2016, MNRAS, 460, 4230

Dolphin A. E. et al., 2001, ApJ, 550, 554

Dyson J. E., de Vries J., 1972, A\&A, 20, 223

Fraser O. J., Hawley S. L., Cook K. H., Keller S. C., 2005, AJ, 129, 768

Fraser O. J., Hawley S. L., Cook K. H., 2008, AJ, 136, 1242

Goldman S. R. et al., 2017, MNRAS, 465, 403

Golshan R. H., Javadi A., van Loon J. Th., Khosroshahi H., Saremi E., 2017, MNRAS, 466, 1764

Groenewegen M. A. T., Whitelock P. A., Smith C. H., Kerschbaum F., 1998, MNRAS, 293, 18

Herrero A., Garcia M., Uytterhoeven K., Najarro F., Lennon D. J., Vink J. S., Castro N., 2010, A\&A, 513, A70

Hidalgo S. L., Marín-Franch A., Aparicio A., 2003, AJ, 125, 1247

Hidalgo S. L., Aparicio A., Gallart C., 2008, AJ, 136, 2332

Hidalgo S. L. et al., 2013, ApJ, 778, 103

Hodge P., 1989, ARA\&A, 27, 139

Humphreys R. M., 1980, ApJ, 238, 65

Iben I., Jr, Renzini A., 1983, ARA\&A, 21, 271

Javadi A., van Loon J. Th., Mirtorabi M. T., 2011, MNRAS, 414, 3394

Javadi A., van Loon J. Th., Khosroshahi H., Mirtorabi M. T., 2013, MNRAS, 432,2824

Javadi A., van Loon J. Th., Khosroshahi H., Tabatabaei F., Golshan R. H., Rashidi M., 2017, MNRAS, 464, 2103

Kirby E. N., Bullock J. S., Boylan-Kolchin M., Kaplinghat M., Cohen J. G., 2014, MNRAS, 439, 1015

Kiss L. L., Szabó G. M., Bedding T. R., 2006, MNRAS, 372, 1721

Kroupa P., 2001, MNRAS, 322, 231

Lagadec E., Zijlstra A. A., 2008, MNRAS, 390, L59

Lake G., Skillman E. D., 1989, AJ, 98, 1274

Maraston C., 2005, MNRAS, 362, 799

Maraston C., Daddi E., Renzini A., Cimatti A., Dickinson M., Papovich C., Pasquali A., Pirzkal N., 2006, ApJ, 652, 85

Marigo P., Girardi L., Bressan A., Groenewegen M. A. T., Silva L., Granato G. L., 2008, A\&A, 482, 883

Marigo P., Girardi L., Bressan A., Rosenfield P., Aringer B., Chen Y., Trabucchi M., 2017, ApJ, 835, 77

Meixner M. et al., 2006, AJ, 132, 2268

Menzies J. W., Whitelock P. A., Feast M. W., 2015, MNRAS, 452, 910

Mo H., van den Bosch F., White S. D. M., 2010, Galaxy Formation and Evolution. Cambridge Univ. Press, Cambridge

Orban C., Gnedin O. Y., Weisz D. R., Skillman E. D., Dolphin A. E., Holtzman J. A., 2008, ApJ, 686, 1030

Pierce M. J., Jurcevic J. S., Crabtree D., 2000, MNRAS, 313, 271

Rezaeikh S., Javadi A., Khosroshahi H., van Loon J. Th., 2014, MNRAS, 445, 2214

Schlegel D. J., Finkbeiner D. P., Davis M., 1998, ApJ, 500, 525

Schröder K. P., Winters J. M., Sedlmayr E., 1999, A\&A, 349, 898

Sibbons L. F., Ryan S., Irwin M., Napiwotzki R., 2015, A\&A, 573, A84

Silich S., Lozinskaya T., Moiseev A., Podorvanuk N., Rosado M., Borissova J., Valdez-Gutierrez M., 2006, A\&A, 448, 123

Skillman E. D., Côté S., Miller B. W., 2003, AJ, 125, 593

Skillman E. D. et al., 2014, ApJ, 786, 44

Soszyński I. et al., 2009, Acta Astron., 59, 239

Stinson G. S., Dalcanton J. J., Quinn T., Kaufmann T., Wadsley J., 2007, ApJ, 667, 170

Tautvaišienė G., Geisler D., Wallerstein G., Borissova J., Bizyaev D., Pagel B. E. J., Charbonnel C., Smith V., 2007, AJ, 134, 2318 
Tikhonov N. A., Galazutdinova O. A., 2002, A\&A, 394, 33

van Loon J. Th., Zijlstra A. A., Whitelock P. A., Waters L. B. F. M., Loup

C., Trams N. R., 1997, A\&A, 325, 585

van Loon J. Th., Cohen M., Oliveira J. M., Matsuura M., McDonald I., Sloan

G. C., Wood P. R., Zijlstra A. A., 2008, A\&A, 487, 1055

Weaver R., McCray R., Castor J., Shapiro P., Moore R., 1977, ApJ, 218, 377

White S. D. M., Frenk C. S., 1991, ApJ, 379, 25

White S. D. M., Rees M. J., 1978, MNRAS, 183, 341

Woods P. M. et al., 2011, MNRAS, 411, 1597

Zijlstra A. A. et al., 2006, MNRAS, 370, 1961

\section{APPENDIX: SUPPLEMENTARY MATERIAL}

In this section, we present figures (Figs A1-A5) and fits (Tables A1A3) to the $K$-band-mass, age-mass, and pulsation duration-mass relation, derived from the Padova models (Marigo et al. 2008, 2017). We used these equations in our analysis to find the SFH of IC 1613. For more detail see Section 3 where we explain the method and present the figures and fits for the case of $Z=$ 0.001 (and $\mu=24.37 \mathrm{mag}$ ). Here we also present isochrones for $Z=0.0007$
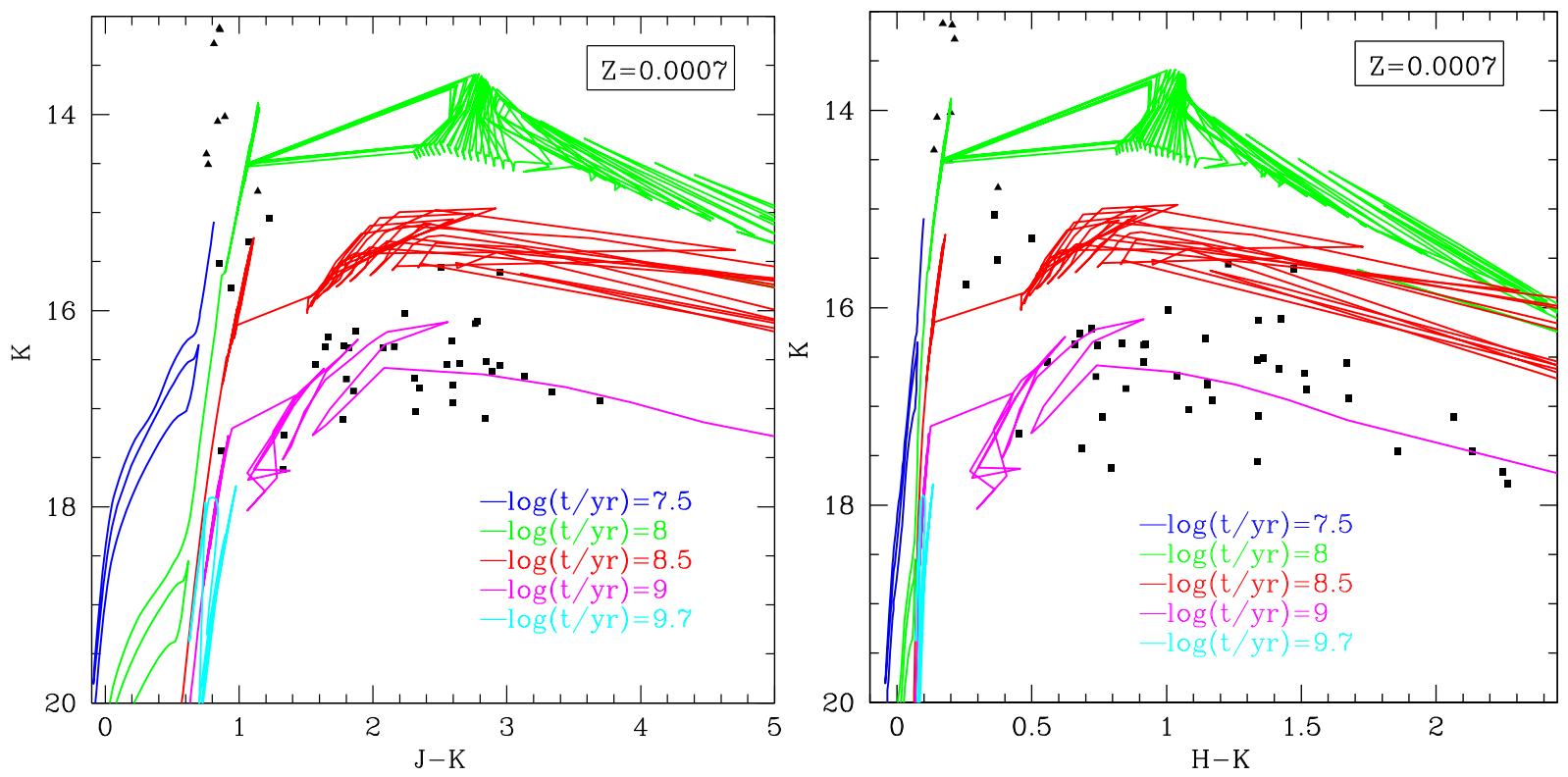

Figure A1. Same as Fig. 2 but for $Z=0.0007$.
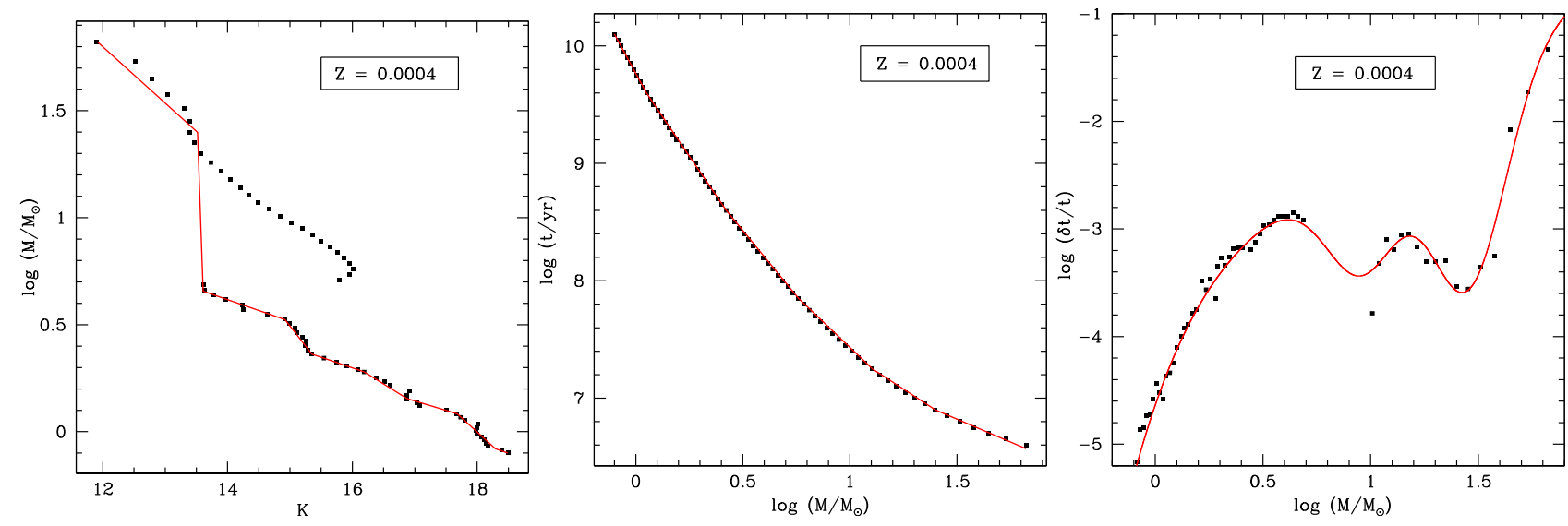

Figure A2. Same as Fig. 4 but for $Z=0.0004$. 

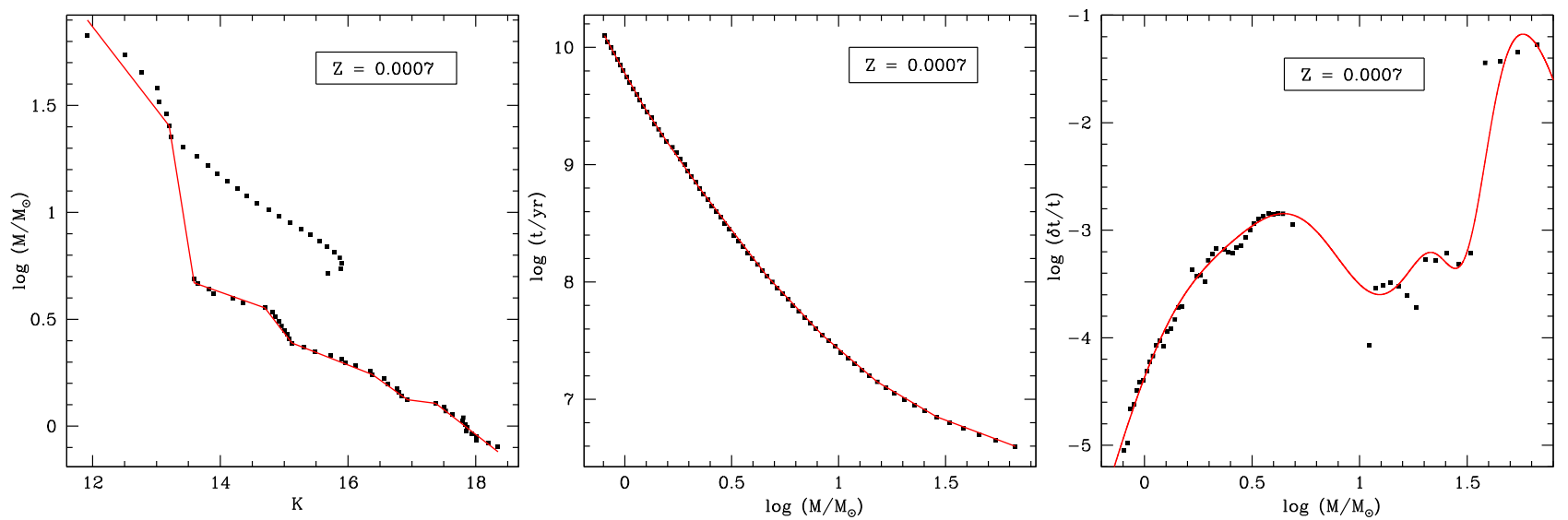

Figure A3. Same as Fig. A2 but for $Z=0.0007$.
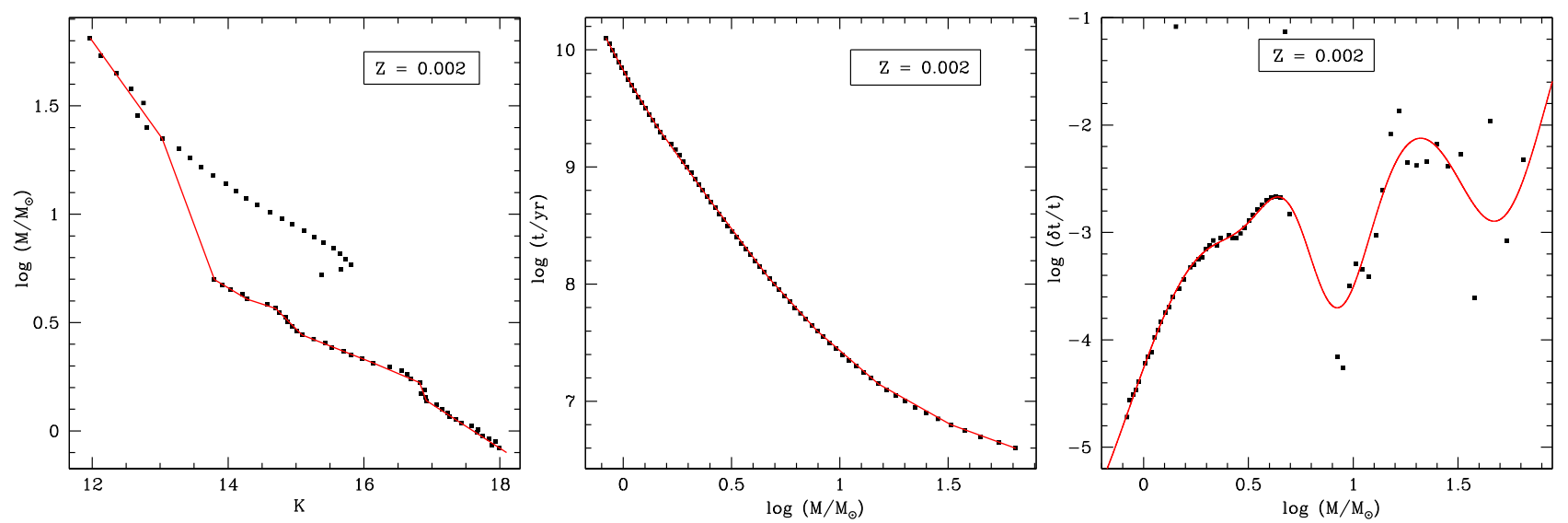

Figure A4. Same as Fig. A2 but for $Z=0.002$.
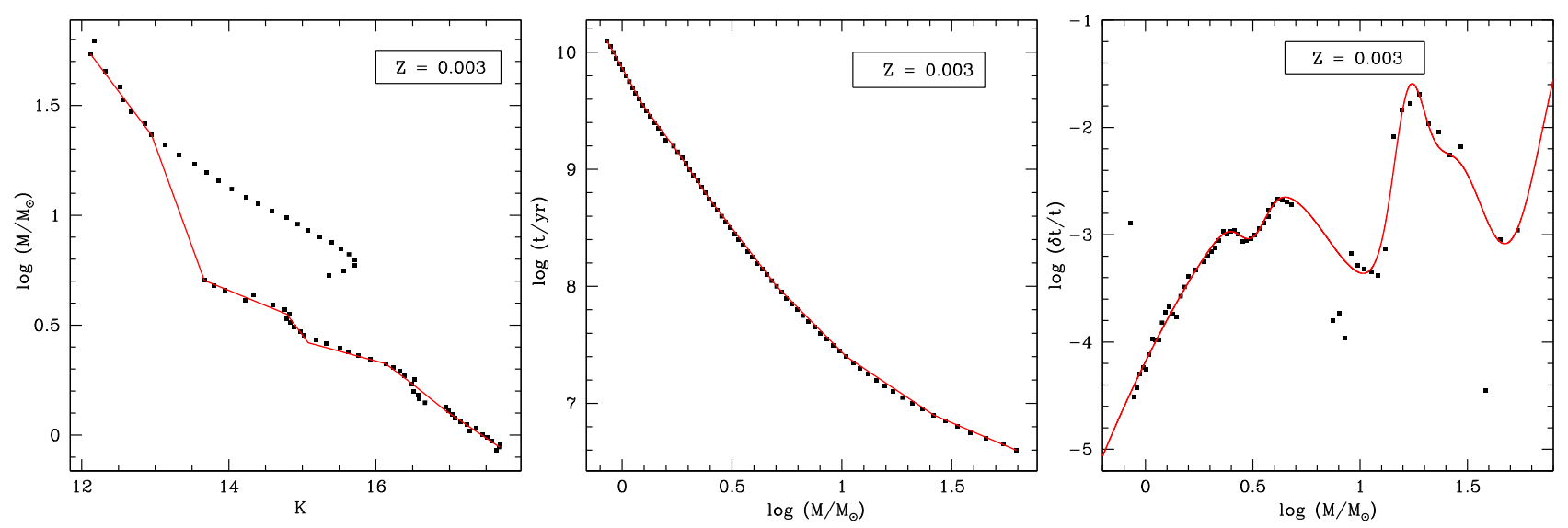

Figure A5. Same as Fig. A2 but for $Z=0.003$. 
Table A1. Fits to the relation between birth mass and $K$-band magnitude, $\log M / \mathrm{M}_{\odot}=a K+b$, for a distance modulus of $\mu=24.37 \mathrm{mag}$.

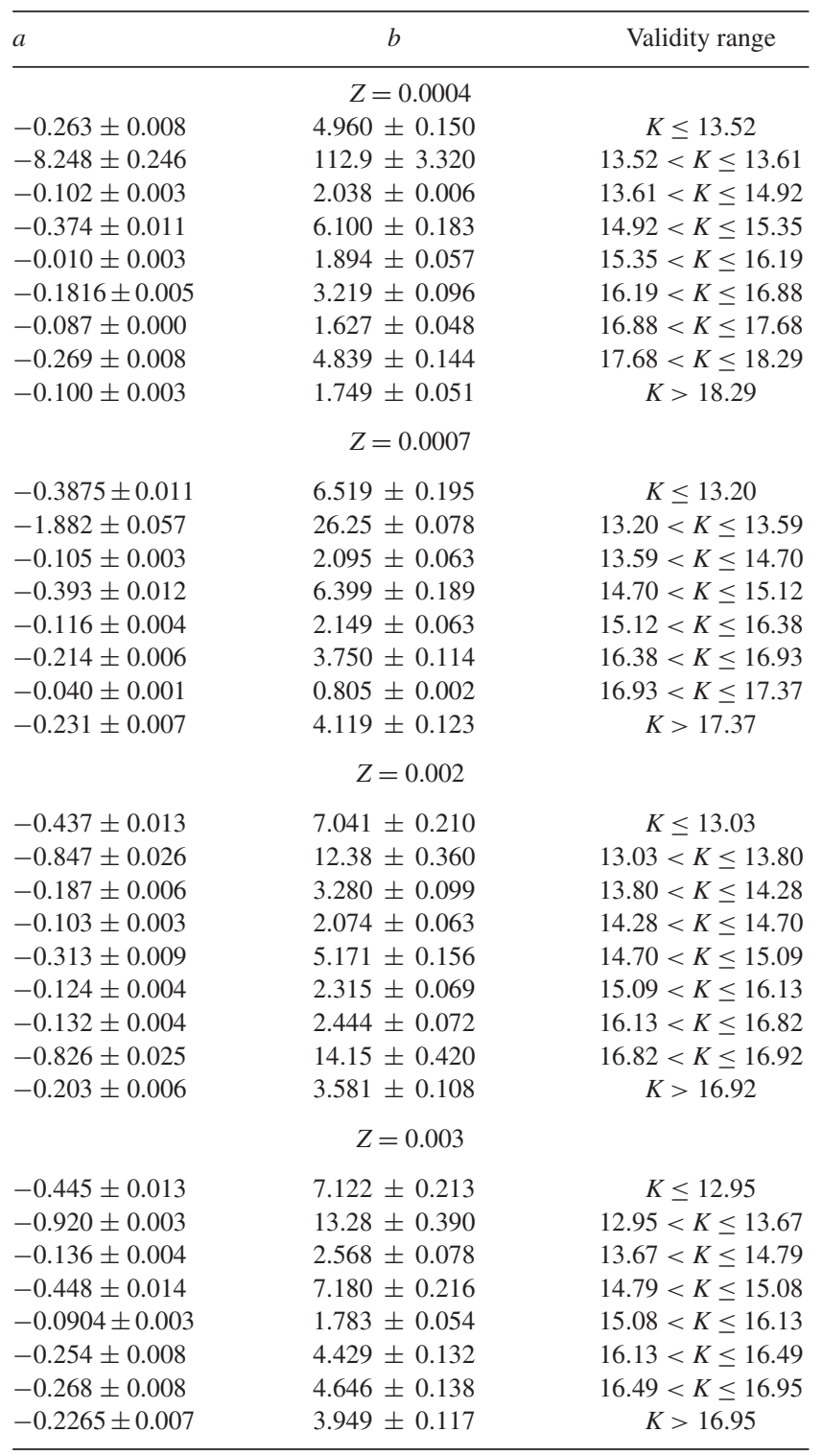

Table A2. Fits to the relation between age and birth mass, $\log t=$ $a \log M / \mathrm{M}_{\odot}+b$.

\begin{tabular}{|c|c|c|}
\hline$a$ & $b$ & Validity range \\
\hline \multicolumn{3}{|c|}{$Z=0.0004$} \\
\hline $\begin{array}{l}-3.333 \pm 0.099 \\
-2.690 \pm 0.081 \\
-2.680 \pm 0.081 \\
-2.225 \pm 0.066 \\
-1.732 \pm 0.051 \\
-1.195 \pm 0.036 \\
-0.778 \pm 0.023\end{array}$ & $\begin{array}{l}9.767 \pm 0.294 \\
9.734 \pm 0.291 \\
9.732 \pm 0.291 \\
9.540 \pm 0.285 \\
9.166 \pm 0.280 \\
8.571 \pm 0.260 \\
7.989 \pm 0.240\end{array}$ & $\begin{array}{c}\log M / \mathrm{M}_{\odot} \leq 0.050 \\
0.050<\log M / \mathrm{M}_{\odot} \leq 0.236 \\
0.236<\log M / \mathrm{M}_{\odot} \leq 0.422 \\
0.422<\log M / \mathrm{M}_{\odot} \leq 0.759 \\
0.759<\log M / \mathrm{M}_{\odot} \leq 1.106 \\
1.106<\log M / \mathrm{M}_{\odot} \leq 1.399 \\
\log M / \mathrm{M}_{\odot}>1.399\end{array}$ \\
\hline \multicolumn{3}{|c|}{$Z=0.0007$} \\
\hline $\begin{array}{l}-3.409 \pm 0.102 \\
-3.191 \pm 0.096 \\
-2.787 \pm 0.084 \\
-2.586 \pm 0.078 \\
-2.376 \pm 0.072 \\
-1.940 \pm 0.057 \\
-1.558 \pm 0.048 \\
-1.076 \pm 0.033 \\
-0.679 \pm 0.020\end{array}$ & $\begin{array}{l}9.780 \pm 0.294 \\
9.781 \pm 0.294 \\
9.745 \pm 0.291 \\
9.706 \pm 0.291 \\
9.632 \pm 0.288 \\
9.342 \pm 0.279 \\
8.989 \pm 0.270 \\
8.419 \pm 0.252 \\
7.841 \pm 0.234\end{array}$ & $\begin{aligned} \log M / \mathrm{M}_{\odot} \leq 0.010 \\
0.010<\log M / \mathrm{M}_{\odot} \leq 0.100 \\
0.100<\log M / \mathrm{M}_{\odot} \leq 0.196 \\
0.196<\log M / \mathrm{M}_{\odot} \leq 0.350 \\
0.350<\log M / \mathrm{M}_{\odot} \leq 0.660 \\
0.660<\log M / \mathrm{M}_{\odot} \leq 0.920 \\
0.920<\log M / \mathrm{M}_{\odot} \leq 1.181 \\
1.181<\log M / \mathrm{M}_{\odot} \leq 1.459 \\
\log M / \mathrm{M}_{\odot}>1.459\end{aligned}$ \\
\hline \multicolumn{3}{|c|}{$Z=0.002$} \\
\hline $\begin{array}{l}-3.412 \pm 0.136 \\
-3.041 \pm 0.090 \\
-2.582 \pm 0.078 \\
-2.431 \pm 0.096 \\
-2.024 \pm 0,060 \\
-1.598 \pm 0.048 \\
-1.045 \pm 0.030 \\
-0.667 \pm 0.020\end{array}$ & $\begin{array}{l}9.827 \pm 0.294 \\
9.819 \pm 0.294 \\
9.748 \pm 0.291 \\
9.689 \pm 0.291 \\
9.415 \pm 0.282 \\
9.033 \pm 0.270 \\
8.381 \pm 0.252 \\
7.809 \pm 0.234\end{array}$ & $\begin{array}{c}\log M / \mathrm{M}_{\odot} \leq 0.020 \\
0.020<\log M / \mathrm{M}_{\odot} \leq 0.150 \\
0.150<\log M / \mathrm{M}_{\odot} \leq 0.380 \\
0.380<\log M / \mathrm{M}_{\odot} \leq 0.670 \\
0.670<\log M / \mathrm{M}_{\odot} \leq 0.890 \\
0.890<\log M / \mathrm{M}_{\odot} \leq 1.170 \\
1.170<\log M / \mathrm{M}_{\odot} \leq 1.510 \\
\log M / \mathrm{M}_{\odot}>1.510\end{array}$ \\
\hline \multicolumn{3}{|c|}{$Z=0.003$} \\
\hline $\begin{array}{l}-3.360 \pm 0.102 \\
-2.523 \pm 0.075 \\
-2.769 \pm 0.084 \\
-2.450 \pm 0.075 \\
-1.892 \pm 0.060 \\
-1.256 \pm 0.039 \\
-0.796 \pm 0.024\end{array}$ & $\begin{array}{l}9.865 \pm 0.347 \\
9.786 \pm 0.030 \\
9.848 \pm 0.294 \\
9.722 \pm 0.291 \\
9.329 \pm 0.279 \\
8.681 \pm 0.261 \\
8.028 \pm 0.240\end{array}$ & $\begin{array}{c}\log M / \mathrm{M}_{\odot} \leq 0.090 \\
0.090<\log M / \mathrm{M}_{\odot} \leq 0.250 \\
0.250<\log M / \mathrm{M}_{\odot} \leq 0.390 \\
0.390<\log M / \mathrm{M}_{\odot} \leq 0.700 \\
0.700<\log M / \mathrm{M}_{\odot} \leq 1.020 \\
1.020<\log M / \mathrm{M}_{\odot} \leq 1.410 \\
\log M / \mathrm{M}_{\odot}>1.410\end{array}$ \\
\hline
\end{tabular}


Table A3. Fits to the relation between the relative pulsation duration and birth mass, $\log (\delta t / t)=\Sigma_{i=1}^{5} a_{i} \exp \left[-\left(\log M\left[\mathrm{M}_{\odot}\right]-b_{i}\right)^{2} / c_{i}^{2}\right]$.

\begin{tabular}{|c|c|c|c|}
\hline$i$ & $a$ & $b$ & $c$ \\
\hline \multicolumn{4}{|c|}{$Z=0.0004$} \\
\hline 1 & -113.2 & -2.296 & 2.073 \\
\hline 2 & -1.084 & 0.969 & 0.239 \\
\hline 3 & 55.97 & -1.295 & 1.580 \\
\hline 4 & -1.963 & 1.456 & 0.248 \\
\hline 5 & 0.000 & 2.385 & 0.022 \\
\hline \multicolumn{4}{|c|}{$Z=0.0007$} \\
\hline 1 & -560.9 & 147.7 & 74.38 \\
\hline 2 & -2.084 & 0.468 & 0.404 \\
\hline 3 & 15.58 & 1.161 & 1.313 \\
\hline 4 & -1.373 & 1.479 & 0.141 \\
\hline 5 & -7.463 & 1.132 & 0.531 \\
\hline \multicolumn{4}{|c|}{$Z=0.002$} \\
\hline 1 & -2.525 & 0.741 & 0.924 \\
\hline 2 & 9.158 & 0.735 & 0.238 \\
\hline 3 & -2.027 & 1.719 & 0.304 \\
\hline 4 & -9.475 & 0.754 & 0.258 \\
\hline 5 & -5.478 & -0.489 & 0.606 \\
\hline \multicolumn{4}{|c|}{$Z=0.003$} \\
\hline 1 & -721.0 & -10.65 & 4.693 \\
\hline 2 & -0.340 & 0.499 & 0.090 \\
\hline 3 & -1.961 & 1.089 & 0.359 \\
\hline 4 & 1.288 & 1.225 & 0.099 \\
\hline 5 & -2.240 & 1.697 & 0.223 \\
\hline
\end{tabular}

This paper has been typeset from a $\mathrm{T}_{\mathrm{E}} \mathrm{X} / \mathrm{LT} \mathrm{E} \mathrm{X}$ file prepared by the author. 